JAQUELINE BARROS LOPES

\title{
Prevalência e fatores de risco para fraturas vertebrais em idosos da comunidade
}

Tese apresentada a Faculdade de Medicina da Universidade de São Paulo para obtenção do título de Doutor em Ciências

Área de concentração: Reumatologia

Orientadora: Profa. Dra. Rosa Maria Rodrigues Pereira

São Paulo

2009 


\section{Dados Internacionais de Catalogação na Publicação (CIP)}

Preparada pela Biblioteca da

Faculdade de Medicina da Universidade de São Paulo

Creprodução autorizada pelo autor

Lopes, Jaqueline Barros

Prevalência e fatores de risco para fraturas vertebrais em idosos da comunidade / Jaqueline Barros Lopes. -- São Paulo, 2009.

Tese (doutorado)--Faculdade de Medicina da Universidade de São Paulo.

Departamento de Clínica Médica.

Área de concentração: Reumatologia.

Orientador: Rosa Maria Rodrigues Pereira.

Descritores: 1.Fraturas da coluna vertebral 2.Idoso 3.Densidade mineral óssea 4.Fêmur 5.Quedas 6.Fatores de risco

USP/FM/SBD-381/09 


\section{DEDICATÓRIAS}

Aos meus pais Francisco Lopes Pereira e Maria José de Barros Lopes pelo dom da vida e, sobretudo, pelos ensinamentos que embasam minha caminhada pelo mundo.

Aos meus irmãos Janine Barros Lopes e Jacques Barros Lopes pelo incentivo e apoio nos momentos difíceis.

Ao meu esposo Rodrigo Favoreto, grande incentivador de meus projetos, pelo carinho, apoio e compreensão diante da minha carga de trabalho e dedicação para que este sonho se tornasse possível. 


\section{AGRADECIMENTOS}

A todos que contribuíram para a realização deste trabalho em especial:

À minha orientadora Profa. Dra. Rosa Maria R. Pereira, por quem tenho grande admiração e respeito, pela amizade, ensinamentos, conselhos, paciência, e acima de tudo, por ter acreditado no meu potencial.

À Camille F. Danilevícius pelo companheirismo, apoio na coleta e compilação dos dados, fundamental para a execução deste projeto.

À Valéria F. Cabarbo pela dedicação, disponibilidade e auxílio em toda logística para aquisição de materiais, preparação dos relatórios e prestação de contas junto a FAPESP.

À Liliam Takayama pela realização das dosagens de vitamina D e PTH, e apoio nas muitas horas trabalhadas.

Ao Hospital Universitário da FMUSP-SP na pessoa do Prof. Dr. Paulo Lotufo, por disponibilizar o serviço para a realização das radiografias e alguns exames laboratoriais incluídos neste projeto.

Ao Prof. Dr. Paulo Rossi Menezes e sua esposa Márcia Scazufca por disponibilizar a equipe de entrevistadores para convidar os idosos ao comparecimento nas avaliações, através do trabalho epidemiológico em conjunto com apoio FAPESP \#04/12694-8.

À profa. Dra. Eloísa Bonfá pela competência e por propiciar um ambiente adequado para o desenvolvimento de pesquisa na disciplina de reumatologia. 
Ao Centro de Estudos em Reumatologia de São Paulo da disciplina de Reumatologia da FMUSP, pelo apoio na realização de análises estatísticas.

À Cláudia Reis de Oliveira, Marta Caggiano, Iná Silva dos Santos e Maria de Fátima Correia da Silva, pela prestatividade com auxílio em todas as vezes que necessitei.

À Maria José (mãe da Camille), fundamental nesses últimos meses, que voluntariamente nos ajudou na organização e arquivo de todos os dados.

À Tatiana Rodrigues da Silva pelo auxílio na organização dos arquivos, coleta e centrifugação das amostras de sangue. Sua agilidade foi essencial para execução deste trabalho.

À Daniela Olegário pela dedicação e realização de grande parte das densitometrias ósseas.

À Michela Peanho pela organização, agilidade e competência, qualidades que indubitavelmente fizeram diferença e contribuíram para o bom andamento deste projeto.

A todos os entrevistadores do Projeto Saúde do Idoso pelo empenho em convidar os idosos a participarem da pesquisa.

Aos idosos da pesquisa pela voluntariedade e compromisso, que mesmo diante de frio ou chuva, compareceram para realização das avaliações. Nada seria possível sem sua presença.

À Deus, hoje e sempre. 
Este trabalho foi apoiado pela Fundação de Amparo à Pesquisa do Estado de São Paulo (FAPESP, \#03/09313-0) e pela Coordenação de Aperfeiçoamento de Pessoal de Nível Superior (CAPES). 
Esta tese está de acordo com as seguintes normas em vigor no momento desta publicação:

Referências: adaptado de International committee of Medical Journals Editors (Vancouver)

Universidade de São Paulo. Faculdade de Medicina. Serviço de Biblioteca e Documentação. Guia de apresentação de dissertações, teses e monografias. Elaborado por Annelise Carneiro da Cunha, Maria Julia de A. L. Freddi, Maria F. Crestana, Marinalva de Souza Aragão, Suely Campos Cardoso, Valeria Vilhena, $2^{a}$. Ed. São Paulo; Serviço de biblioteca e Documentação, 2005.

Abreviaturas dos títulos dos periódicos de acordo com List of Journals Indexed In Index Medicus. 


\section{SUMÁRIO}

Lista de abreviaturas

Lista de Figuras, Tabelas e Gráficos

Resumo

Summary

1 INTRODUÇÃO

2 OBJETIVOS

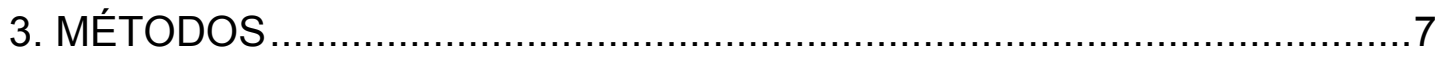

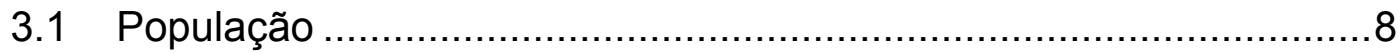

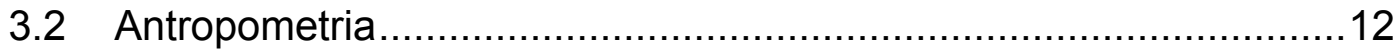

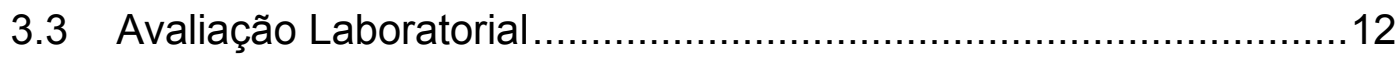

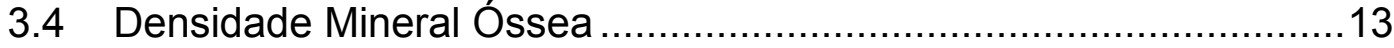

3.5 Avaliação de Fraturas Vertebrais ..................................................14

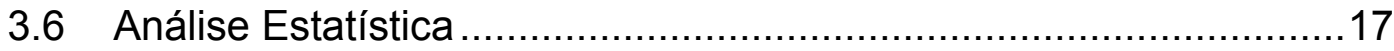

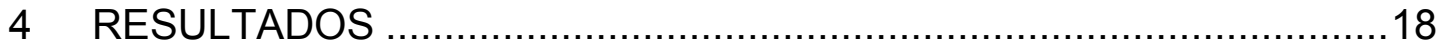

4.1 Características da população estudada .......................................19

4.2 Avaliação das fraturas vertebrais ...............................................24

4.3 Avaliação dos fatores de risco para fraturas vertebrais por

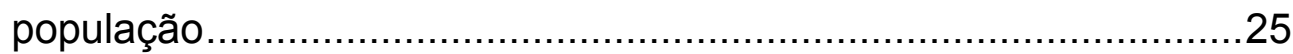

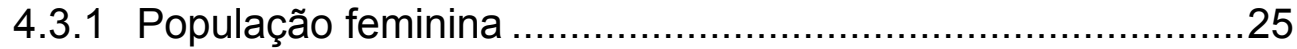

4.3.2 População Masculina.......................................................... 30

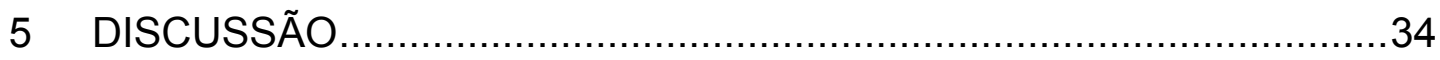

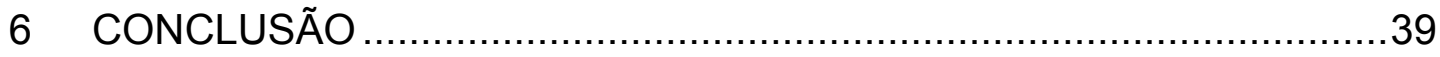

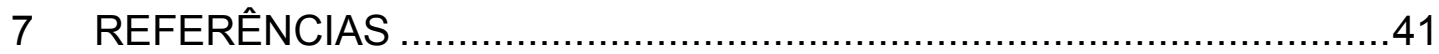

Apêndices 


\section{LISTA DE SIGLAS}

\begin{tabular}{ll} 
DMO & Densidade Mineral Óssea \\
$25 O H D$ & 25-Hidroxivitamina D \\
CV & Coeficiente de Variação \\
DXA & $\begin{array}{l}\text { Dual X-ray Absorptiometry (Dupla Absorciometria de } \\
\text { Raios-X }\end{array}$ \\
eTFG & estimativa da Taxa de Filtração Glomerular \\
FMUSP & Faculdade de Medicina da Universidade de São Paulo \\
IC & Intervalo de Confiança \\
IMC & Índice de Massa Corpórea \\
IOF & Internacional Osteoporosis Foundation \\
ISCD & Internacional Society Clinical e Densitometry \\
LIM & Laboratório de Investigação Médica \\
OMS & Organização Mundial de Saúde \\
OR & Odds ratio (Razão de chance) \\
PTHi & Paratormônio intacto \\
SQ & Semi-quantitativa \\
VMS & Variação Mínima Significativa \\
Vs. & Versus \\
\hline
\end{tabular}




\section{LISTA DE TABELAS}

Tabela 1. Características demográficas, antropométricas e clínicas associadas com osteoporose/fratura e outras co-morbidades na amostra estudada

Tabela 2. Dados laboratoriais, densidade mineral óssea (DMO) nas regiões de coluna lombar, colo femoral e fêmur total na amostra da amostra

Tabela 3. Prevalência (95\% intervalo de confiança) das Fraturas vertebrais por faixa etária e por gênero

Tabela 4. Dados demográficos, antropométricos e clínicos da amostra feminina distribuídos no grupo Fratura Vertebral e Sem Fratura Vertebral.

Tabela 5. Dados laboratoriais e da densidade mineral óssea (DMO) nas regiões de coluna lombar, colo femoral, fêmur total, da amostra feminina distribuídos nos grupos Fratura Vertebral e Sem Fratura Vertebral.

Tabela 6. Análise de regressão logística para a presença de fraturas vertebrais moderada/grave na população feminina

Tabela 7. Dados demográficos, antropométricos e clínicos da amostra masculina distribuídos nos grupos Fratura Vertebral e Sem Fratura Vertebral.

Tabela 8. Dados laboratoriais e da densidade mineral óssea (DMO) nas regiões de coluna lombar, colo femoral, fêmur total, da amostra masculina distribuída nos dois grupos Fratura Vertebral e Sem Fratura Vertebral ....

Tabela 9. Análise de regressão logística para a presença de fraturas vertebrais moderada/grave na amostra masculina 


\section{LISTA DE FIGURAS}

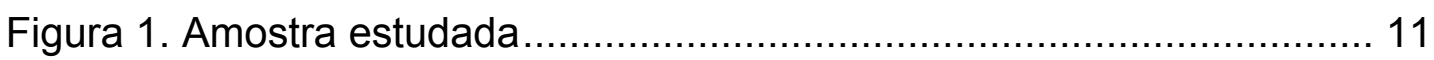

Figura 2. Fratura vertebral moderada (grau 2) .................................... 16

Figura 3. Fratura vertebral grave (grau 3) ...................................... 16 


\section{RESUMO}

Lopes JB. Prevalência e fatores de risco para fraturas vertebrais em idosos da comunidade [tese]. São Paulo: Faculdade de Medicina, Universidade de São Paulo; 2009. 49p.

Objetivo: Estimar a prevalência de fraturas vertebrais investigando os fatores de risco associados com esta última condição em idosos brasileiros da comunidade. Métodos: Este estudo de corte transversal incluiu 769 indivíduos idosos com 65 anos ou mais (462 mulheres e 307 homens) residentes em São Paulo, Brasil. Radiografias de coluna torácica e lombar foram obtidas e fraturas vertebrais foram avaliadas usando o método semiquantitativo de Genant. Densidade mineral óssea (DMO) foi mensurada por DXA e parametros bioquímicos foram também avaliados. Mulheres e homens foram analisados separadamente, e cada gênero foi dividido em 2 grupos com base na presença de fraturas vertebrais. Resultados: A prevalência de fraturas vertebrais foi de $16,7 \%(95 \% \mathrm{Cl} 13,3-20,1)$ nas mulheres e $21,2 \%(95 \% \mathrm{Cl} 16,6-25,7)$ nos homens. Análise de regressão logística usando as variáveis significantes na análise univariada no grupo feminino mostrou que a idade (OR=1,12,95\% Cl 1,06-1,18; $p<0,001)$ e o Tscore do colo femoral $(\mathrm{OR}=0,61,95 \% \mathrm{Cl} 0,46-0,88 ; p=0,006)$ foram fatores de risco independentes na predição de fraturas vertebrais. No grupo masculino, a análise de regressão logística demonstrou que a condição de caidor crônico (OR=2,54 95\% Cl 1,1-5,9; $p=0,031)$ e T-score do colo femoral (OR=0,72, 95\% Cl 0,53-0,96; $p=0,025)$ foram independentes parâmetros na predição de fraturas vertebrais. Conclusão: Nossos resultados sugerem que fraturas vertebrais são comuns em idosos brasileiros e que o T-score do colo femoral baixo foi um importante fator de risco para esta condição em ambos homens e mulheres. Idade também foi significantemente correlacionada com a presença de fraturas vertebrais em mulheres, e a condição de caidor crônico foi correlacionada com fraturas vertebrais em homens.

Descritores: 1.Fraturas vertebrais 2.Idoso 3.Densidade mineral óssea 4.Fêmur 5.Queda 


\section{SUMMARY}

Lopes JB. Prevalence and risk factors of vertebral fractures in communitydwelling elderly [thesis]. São Paulo: "Faculdade de Medicina, Universidade de São Paulo"; 2009. 49p.

Purpose: To estimate the prevalence of radiographic vertebral fracture and investigate factors associated with this condition in Brazilian communitydwelling elderly. Methods: This cross sectional study included 769 elderly subjects 65 years old and over (462 women and 307 men) living in São Paulo, Brazil. Thoracic and lumbar spine radiographs were obtained and vertebral fractures were evaluated using Genant's semi-quantitative method. Bone mineral density (BMD) was measured by DXA and bone biochemical markers were also evaluated. Female and male subjects were analyzed independently, and each gender was divided into 2 groups, based on whether vertebral fractures were present. Results: The prevalence of vertebral fracture was $16.7 \%(95 \% \mathrm{Cl} 13.3-20.1)$ in women and $21.2 \%$ (95\% $\mathrm{Cl}$ 16.6-25.7) in men. Logistic regression analyses using variables that were significant in the univariate analysis, in female group showed that age $(\mathrm{OR}=1.12,95 \% \mathrm{Cl} 1.06-1.18 ; \mathrm{p}<0.001)$ and femoral neck $\mathrm{T}$-score $(\mathrm{OR}=0.61$, $95 \% \mathrm{Cl}$ 0.46-0.88; $\mathrm{p}=0.006$ ) were independently factors in predicting vertebral fracture. In the male group, logistic regression analyzes demonstrated that chronic faller condition $(\mathrm{OR}=2.5495 \% \mathrm{Cl} 1.1-5.9$; $\mathrm{p}=0.031)$ and femoral neck T-score $(\mathrm{OR}=0.72,95 \% \mathrm{Cl} 0.53-0.96 ; \mathrm{p}=0.025)$ were independent parameters in predicting vertebral fractures. Conclusions: Our results suggest that radiographic vertebral fractures are common in Brazilian community-dwelling elderly and that a low femoral neck T-score was an important risk factor for this condition in both males and females. Age was also significantly correlated with the presence of vertebral fractures in women, and chronic faller was correlated with vertebral fractures in men.

Descriptors: 1.Vertebral fracture 2.Elderly people 3.Bone mineral density 4.Femur 5.Fall 


\section{INTRODUÇÃO}


A osteoporose é definida como uma doença esquelética sistêmica caracterizada por baixa densidade mineral óssea (DMO) e comprometimento da resistência óssea levando a fragilidade óssea e conseqüente aumento no risco de fraturas (Consensus Development Conference, 1993).

Dentre as fraturas osteoporóticas, as fraturas vertebrais constituem as mais freqüentes (Melton et al.,1989) e possuem importantes implicações clínicas (Hasserius et al., 2005; Ismail et al., 2001, Delmas et al., 2003).

Fraturas vertebrais prévias estão associadas ao aumento de risco de novas fraturas sejam elas vertebrais ou não (Ismail et al., 2001), especialmente se a fratura vertebral for grave (Delmas et al., 2003). De fato, recentemente, Cauley (2007) encontrou que mulheres com fraturas vertebrais prévias apresentavam um risco quatro vezes maior de novas fraturas em 15 anos de seguimento quando comparadas aquelas sem esta condição (Cauley et al., 2007).

Hasserius (2005) demonstrou que o diagnóstico de fraturas vertebrais em pessoas idosas esteve relacionado com o aumento da morbidade, especialmente em mulheres, e da mortalidade em ambos os gêneros (Hasserius et al., 2005).

Com o crescimento da população de idosos, o impacto das fraturas vertebrais na saúde pública tende a aumentar, notadamente em populações de países em desenvolvimento, onde se calcula que esta população 
aumentará cerca de nove vezes ate 2050, ou seja, de 171 milhões para 1,6 bilhões (United Nations Population Division, 1998).

Apesar desta importância, o subdiagnóstico e o conseqüente não tratamento das fraturas vertebrais são grandes problemas em todo o mundo (Delmas et al., 2005). Estudos epidemiológicos estimaram que a incidência de todas as fraturas vertebrais seja cerca três vezes maior que as fraturas de quadril, mas somente um terço destas fraturas recebe atenção médica (Cooper et al., 1992).

O método de avaliação de fratura vertebral padronizado é a radiografia de coluna torácica e lombar, entretanto, muitas técnicas para interpretação destas radiografias têm sido desenvolvidas, incluindo a técnica semiquantitativa de Genant (Genant et al., 1993). Esta última tem sido escolhida por muitos estudos de osteoporose (Black et al., 1993; Harris et al., 1999; Siris et al., 2002; Reginster et al., 2005; Samelson et. al., 2006) e tornou-se uma ferramenta prática subsidiando diretrizes (Ferrar et al., 2005).

A prevalência das fraturas vertebrais e o estudo dos fatores de risco associados, incluindo avaliação da DMO e de parâmetros laboratoriais não têm sido bem caracterizados em indivíduos saudáveis e assintomáticos, principalmente em populações de países em desenvolvimento como o Brasil (Pinheiro et al., 2009; Clark et al., 2009).

Com relação aos indivíduos latinoamericanos, existe um estudo que analisou a prevalência de fraturas vertebrais exclusivamente em mulheres (Clark et al., 2009). Contudo, tal estudo não foi desenhado especificamente para caracterizar os fatores de risco e não incluiu a avaliação da densidade 
mineral óssea e de parâmetros laboratoriais. Este estudo, também não utilizou a técnica de Genant para detecção das fraturas vertebrais.

Dentro deste contexto, o objetivo do presente trabalho foi determinar a prevalência de osteoporose e de fraturas vertebrais por radiografia usando a técnica semiquantitativa de Genant em indivíduos da comunidade com 65 anos ou mais e avaliar os fatores de risco associados a estas fraturas, incluindo a análise da DMO e parâmetros laboratoriais. 
2 OBJETIVOS 
Estimar a prevalência de fraturas vertebrais em uma população de idosos residentes na área do Butantã.

Verificar a associação das fraturas vertebrais moderadas e graves com:

- fatores de risco para osteoporose (idade, idade da menopausa, tabagismo, alcoolismo, atividade física, ingestão de cálcio, história familiar de fratura, história de fratura por fragilidade);

- parâmetros laboratoriais (25 hidroxivitamina D, PTH, cálcio, fósforo, fosfatase alcalina e creatinina);

- valores da DMO da coluna lombar, fêmur total e colo femural. 
3. MÉTODOS 


\subsection{População}

Este é um estudo de corte transversal com base populacional (Estudo Saúde do Idoso) conduzido de Julho/2005 a Setembro/2007 com idosos da comunidade maiores de 65 anos procedentes da cidade de São Paulo (uma região subtropical, latitude $\left.23^{\circ} 32^{\prime} 51^{\prime \prime}\right)$.

Esta cidade tem uma população de 10,4 milhões de habitantes (IBGE, Instituto Brasileiro de Geografia e Estatística, 2006), e está dividida em 31 distritos administrativos, com demarcação populacional e territorial. A presente investigação foi realizada no distrito Butantã, localizado no lado oeste da cidade. Em 2000 esta área tinha 377.576 residentes, dos quais $6,2 \%$ tinham 65 anos ou mais. O estudo foi conduzido em 66 setores (distritos administrativos menores) compreendendo uma população de aproximadamente 63.000 residentes, representando $17 \%$ da população total do distrito Butantã. A seleção destas áreas foi baseada nas equipes de programa de Saúde da Família, mas não limitado por elas. Esta região possui as mesmas características de distribuição de idade, gênero e classe social que a população de idosos do Brasil (IBGE, 2002)

Entrevista individual foi realizada utilizando um questionário específico para obter informações sobre saúde e estilo de vida, com atenção especial aos fatores de risco para osteoporose/fraturas conforme estudos prévios 
(O’Neill et al., 1995; O'Neill et al., 1996). Estes fatores incluíram: atividade física, etilismo, tabagismo atual, uso de glicorticóide, história familiar de fratura de quadril após os 50 anos em parentes de primeiro grau, história de queda no último ano, ingestão de cálcio, idade da menopausa e presença de dorsalgia.

Atividade física nos últimos 12 meses foi classificada como: a) baixa, quando o individuo não realizava nem atividades domésticas; b) moderada, quando o individuo fazia regularmente as atividades domésticas e/ou caminhadas irregulares; c) alta, quando o indivíduo realizava regularmente atividade física, além de sua atividade de rotina diária, no mínimo duas vezes na semana por 30 minutos (Fitti et al., 1987).

Tabagismo atual, etilismo, uso de glicocorticóide, história familiar de fratura de quadril e dorsalgia foram medidas como variáveis binárias.

Etilismo foi considerado positivo se a ingestão atual de álcool era maior ou igual a três unidades de álcool/dia. O uso de glicocorticóide foi considerado positivo se, no momento da entrevista, o indivíduo estivesse fazendo uso de prednisona ou equivalente na dose de $5 \mathrm{mg} / \mathrm{dia}$ ou mais por no mínimo três meses consecutivos.

Indivíduos que apresentaram duas ou mais quedas nos últimos 12 meses foram definidos como caidores crônicos (Schwartz et al., 1999). Fratura por fragilidade foi definida como aquela ocorrida após 50 anos de idade, decorrente de queda da própria altura ou trauma menor em sítios típicos de fragilidade óssea (quadris, antebraço, punho, úmero, fêmur, etc). Fraturas ocorridas em tornozelo, crânio, face, falanges e dedos do pé não foram consideradas como fraturas osteoporóticas por fragilidade (Seeley et al., 1991). 
A ingestão de cálcio foi estimada a partir da ingestão de leite e derivados. Os participantes responderam se bebiam leite regularmente, comiam queijo ou tomavam iogurte. Se a resposta era positiva, eles foram solicitados a quantificar a média de consumo (baseada nos últimos três dias antes da entrevista) de leite ou iogurte $(\mathrm{mL} / \mathrm{d})$ e queijo $(\mathrm{g} / \mathrm{d})$.

A etnia foi definida baseada na cor relatada por cada participante de acordo com os seus ancestrais até a segunda geração, como validado previamente para a população brasileira (Fuchs et al., 2002). Aqueles que relataram os quatro avôs brancos foram classificados como brancos. A presença de brancos e negros, ou mulatos, nos ascendentes determinava a classificação como não-branca. Na ausência de informações sobre os avôs, a etnia foi determinada pela raça de seus pais. Descendentes de outras raças não foram incluídas.

No total foram 1368 indivíduos convidados e 1025 (385 homens e 640 mulheres) submeteram-se a todas avaliações. Dos 1025 avaliados, $123(12 \%)$ foram excluídos: 70 usando drogas que afetavam o metabolismo ósseo (bisfosfonatos, anticonvulsivantes, vitamina D e/ou cálcio suplementar), 20 por neoplasia prévia (< cinco anos) ou atual com possibilidade de metástase óssea, 15 por apresentarem hiperparatireoidismo primário (cálcio sérico > 10,1 mg/dL e PTH > 62 pg/mL), 12 por insuficiência renal (creatinina $>$ 1,5 mg/dL), 06 por apresentarem artrite reumatóide (Figura 1).

Todos os idosos incluídos eram independentes e não apresentavam evidência de má absorção, diarréia crônica, doença hepática, câncer ou outras doenças crônicas determinantes de uma baixa expectativa de vida. 
O estudo foi aprovado pelo comitê de ética local e o termo de consentimento livre e esclarecido foi obtido de todos os participantes por escrito.

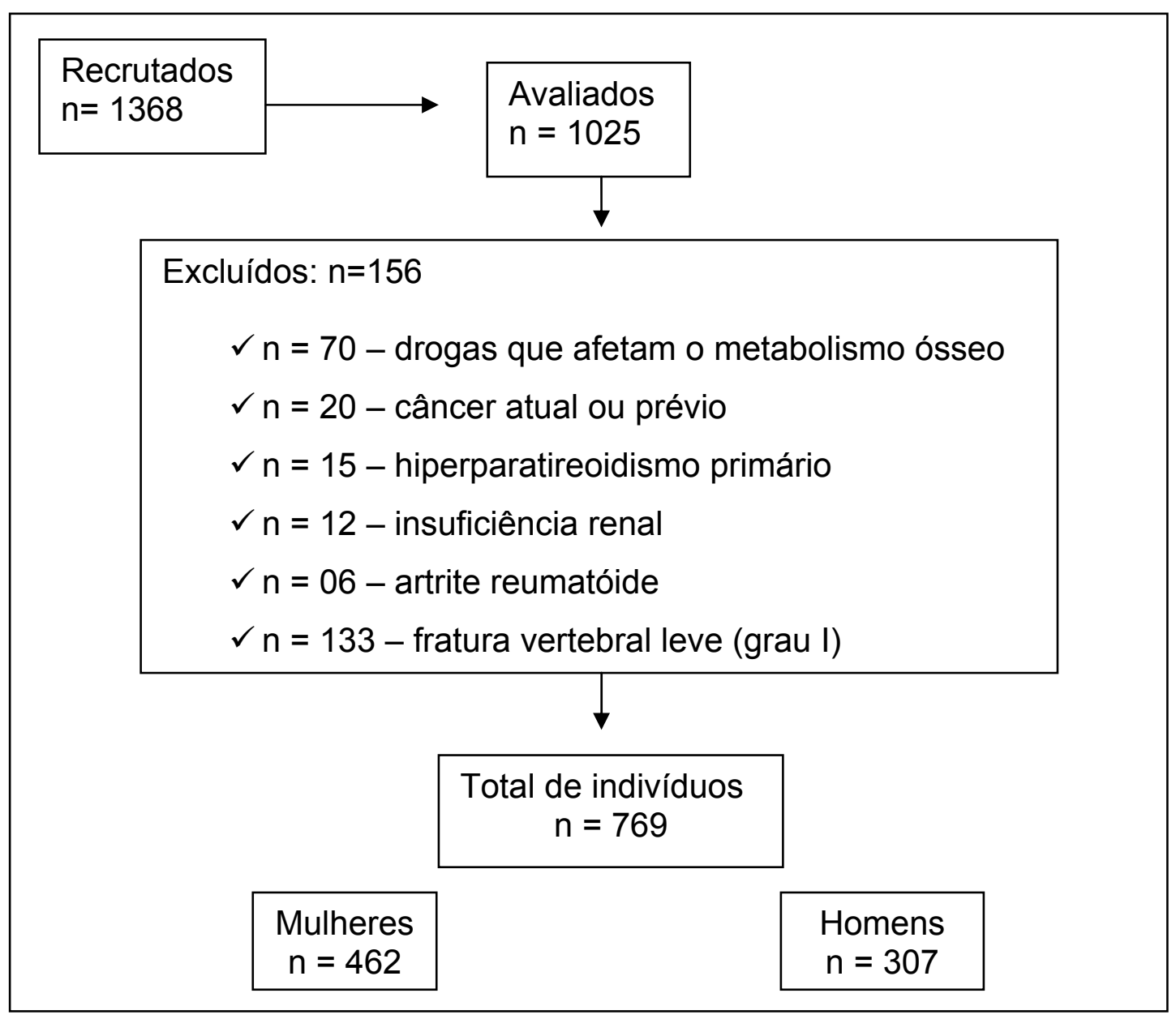

Figura 1. Amostra estudada 


\subsection{Antropometria}

Para avaliação antropométrica, todos os participantes usavam roupas leves e estavam sem sapatos. A altura e o peso foi medida dentro de $0,1 \mathrm{~cm}$ e 0,25 kg com um estadiômetro de parede e uma balança de trave dupla, respectivamente. O índice de massa corpórea (IMC) foi calculado pelo peso em kilogramas, dividido pela altura ao quadrado em metros.

\subsection{Avaliação Laboratorial}

Amostras de sangue foram coletadas em jejum, entre 08h00min e 10h00min da manhã. As dosagens séricas de cálcio, fósforo, fosfatase alcalina e creatinina foram realizadas pela rotina no Hospital Universitário com métodos padronizados. A estimativa da taxa da filtração glomerular (eTFG) foi calculada usando a equação de Cockroft-Gault (Cockcroft et al., 1976).

Uma alíquota de sangue foi estocada a $-70^{\circ} \mathrm{C}$ no Laboratório de Metabolismo Ósseo da Reumatologia (Laboratório de Investigação MédicaLIM17) para posterior dosagem de 25 hidroxivitamina D (25OHD) e paratormônio intacto (PTHi).

A concentração sérica de $250 \mathrm{HD}$ foi determinada através da técnica de radioimunoensaio (DiaSorin, Stillwater, Minnesota, EUA). O menor limite de detecção deste ensaio era de $5 \mathrm{ng} / \mathrm{mL}$. Os coeficientes de variação $(\mathrm{CV})$ 
intra-ensaio e interensaio em nosso laboratório foram de 10,5\% e 17,8\%, respectivamente.

A concentração sérica de PTHi foi mensurada por ensaio imunoradiométrico (ELSA-PTH, CIS bio international, França), com referência de variação de11-62 pg/mL. Os coeficientes de variação (CV) intra-ensaio e interensaio foram de 2,5\% e 6,1\%, respectivamente.

\subsection{Densidade Mineral Óssea}

A densidade mineral óssea (DMO) foi realizada na Faculdade de Medicina da Universidade de São Paulo (FMUSP), sendo mensurada por dupla absorciometria de raios-X (DXA) usando o equipamento de densitometria Hologic (Hologic Inc, Bedford, MA, USA, modelo Discovery) nas regiões: coluna lombar, colo femoral e fêmur total. Todas as medidas de DMO foram realizadas por tecnólogo treinado.

A DMO, expressa em $\mathrm{g} / \mathrm{cm}^{2}$, foi determinada na região de coluna lombar L1-L4, colo femoral e fêmur total. O erro de precisão para estas medidas foi determinado de acordo com a padronização da International Society for Clinical Densitometry (ISCD) (Shepherd et al., 2006). A variação mínima significativa (VMS) com intervalo de confiança de $95 \%$ foi 0,033 $\mathrm{g} / \mathrm{cm}^{2}$ para coluna lombar, $0,047 \mathrm{~g} / \mathrm{cm}^{2}$ para o colo femoral e $0,039 \mathrm{~g} / \mathrm{cm}^{2}$ para o fêmur total. 
Utilizando o menor T-score entre os três sítios (coluna lombar, colo femoral e fêmur total), cada participante foi classificado em osteoporose, osteopenia ou normal. De acordo com a OMS, o indivíduo que apresenta DMO menor ou igual a $-2,5$ desvios padrão em relação a controles saudáveis no pico de massa óssea ( $T$-score) têm o diagnóstico de osteoporose; o indivíduo entre -2,5 e -1,0 desvios padrão ( $T$-score) tem osteopenia e são normais os acima de $-1,0$ desvios padrão ( $T$-score) (Anonymous WHO Study, 1994).

\subsection{Avaliação de Fraturas Vertebrais}

Os participantes foram colocados na posição lateral esquerda e as radiografias da coluna vertebral torácica e lombar obtidas com 40" de distância tubo-filme de acordo com o padronizado para a técnica radiográfica utilizada. Radiografias da coluna torácica foram centradas em T7 e da coluna lombar em L2. Todas as radiografias adquiridas foram de boa qualidade para a análise das vértebras do seguimento T4 a L4.

A detecção das fraturas vertebrais foi realizada por decisão concomitante de dois avaliadores com experiência na área. Para tal, utilizou-se a técnica semiquantitativa (SQ) de Genant (Genant et al., 1993) onde, cada vértebra da região T4-L4 foi avaliada para decidir se havia alguma fratura. 
Cada vértebra julgada como fratura foi graduada de acordo com a escala SQ de Genant. Assim, fratura leve (grau 1) foi definida como redução de $20-25 \%$ da altura anterior, média ou posterior; fratura moderada (grau 2) (Figura 2), como uma redução de $26-40 \%$ da altura vertebral e fratura grave (grau 3) (Figura 3), como uma redução acima de 40\% (Genant et al., 1993).

Como vários estudos epidemiológicos definem fraturas vertebrais apenas as classificadas como moderadas (grau 2) ou graves (grau 3), uma vez que as fraturas leves (grau 1) podem ser secundárias a deformidades pela idade e/ou osteoartrite (Ferrar et al., 2007; El Maghraoui et al., 2008); 113 indivíduos apresentando apenas fraturas leves (grau I) foram excluídos do estudo.

A prevalência de fraturas vertebrais foi definida considerando a presença de pelo menos uma fratura vertebral moderada ou grave. De acordo com a presença ou ausência destas fraturas vertebrais nas radiografias os participantes foram classificados em dois grupos: Fratura Vertebral - indivíduos com ao menos uma fratura vertebral moderada ou grave; Sem Fratura Vertebral - indivíduos sem fratura vertebral 


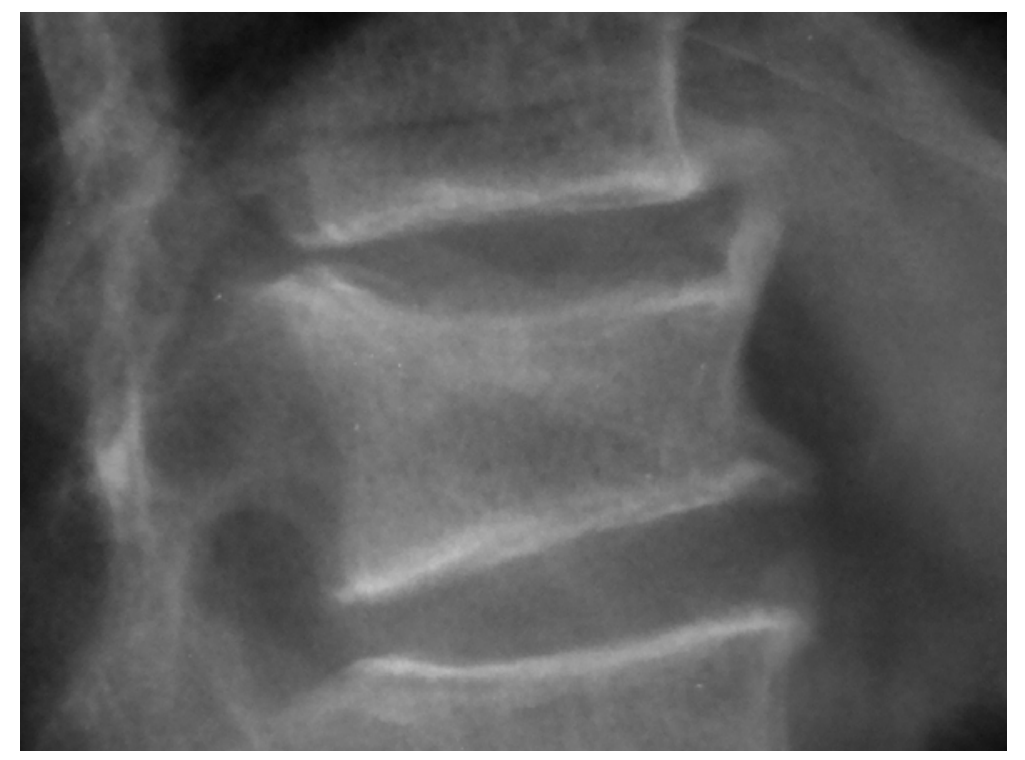

Figura 2. Fratura vertebral moderada (grau 2)

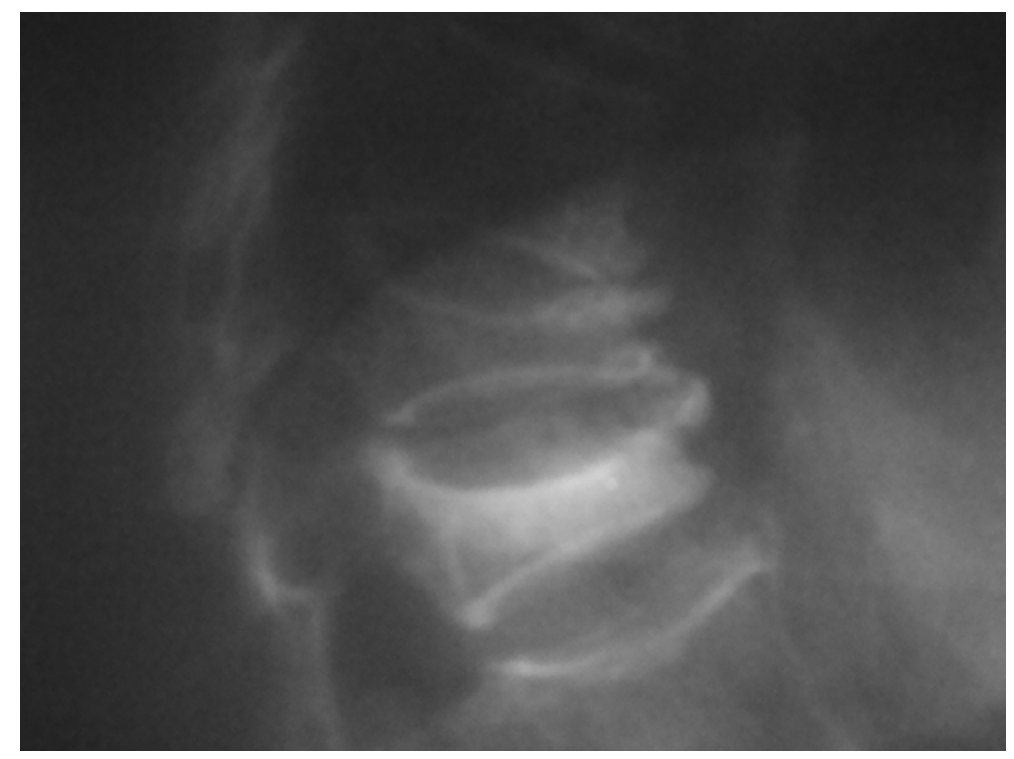

Figura 3. Fratura vertebral grave (grau 3) 


\subsection{Análise Estatística}

Os resultados foram expressos em média \pm desvio padrão ou porcentagens. Análise univariada foi realizada usando t-Student, MannWhitney $U$ ou qui-quadrado. Variáveis com associação significativa $(P \leq 0,05)$ na análise univariada foram incluídas em modelos de regressão logística para predizer os fatores mais importantes relacionados à presença Fraturas vertebrais moderada/grave e de fraturas não vertebrais. Modelos de regressão logística foram criados com inclusão individual das DMO de cada sítio analisado. Os resultados foram apresentados com odds ratios (OR) ajustados correspondendo a 95\% de intervalo de confiança (IC). As análises foram obtidas através do programa Stata 9,0. 
4 RESULTADOS 


\subsection{Características da população estudada}

A idade da população feminina desta amostra variou de 65 a 93 anos com média de idade de 73,5 $\pm 5,4$ anos e as seguintes características antropométricas: peso $65,4 \pm 13,4 \mathrm{~kg}$, altura de $150,5 \pm 6,1 \mathrm{~cm}$ e IMC de $28,8 \pm 5,4 \mathrm{Kg} / \mathrm{m}^{2}$. A população masculina teve idade variando de 66 a 92 anos, com média de idade de $72,7 \pm 5,0$ anos e valores antropométricos de: peso 70,7 \pm 12,3 kg; altura 162,7 \pm 7,6 cm e IMC 26,6 $\pm 3,9 \mathrm{Kg} / \mathrm{m}^{2}$ (Tabela 1).

Dentre as mulheres, $304(65,8 \%)$ foram classificadas como brancas e $158(34,2 \%)$ como não brancas. Dos homens, $257(69,1 \%)$ eram descendentes de brancos, 109 (30,9\%) de não brancos (Tabela 1).

A média de idade da menopausa foi de 46,1 $\pm 10,9$ anos (Tabela 1). Etilismo atual foi relatado por apenas duas $(0,4 \%)$ mulheres e por $32(10,4 \%)$ homens. Em relação à história de tabagismo, 46 (10\%) mulheres e 47 $(15,3 \%)$ homens referiram tabagismo atual (Tabela 1$)$.

O uso de glicocorticóide por mais de três meses consecutivos, no momento da análise, foi detectado em $8(1,7 \%)$ das mulheres e $3(1,0 \%)$ dos homens (Tabela 1), sendo que as causas para o uso do mesmo foram asma e doença pulmonar obstrutiva crônica.

História de fratura por fragilidade foi descrita em 74 (16,0\%) mulheres e em $18(5,9 \%)$ homens. Nenhum indivíduo relatou possuir fratura vertebral 
durante a entrevista. História familiar de fratura de quadril foi mencionada por $42(9,9 \%)$ mulheres e $18(6,6 \%)$ homens (Tabela 1). Entretanto, $85(9,4 \%)$ idosos foram impossibilitados de responder por falecimento dos pais ainda jovens (antes dos 50 anos de idade), correspondendo a $39(8,4 \%)$ das mulheres e $34(11 \%)$ homens.

Foram considerados caidores crônicos 77 mulheres (16,6\%) e 28 (9,1\%) homens. Verificaram-se valores baixos de ingestão de cálcio nas duas populações, com média de $445 \pm 311 \mathrm{mg} /$ dia entre as mulheres e de entre os homens $409 \pm 320 \mathrm{mg} / \mathrm{dia}$ (Tabela 1 ).

Quarenta e seis $(10,0 \%)$ mulheres relataram atividade física baixa, $312(67,5 \%)$ atividade física moderada e 104 (22,5\%) atividade física alta. Os homens apresentaram padrão de atividade física semelhante. Entre as doenças mais relatadas observou-se hipertensão arterial sistêmica (HAS) e diabetes mellitus (DM) (Tabela 1). 
Tabela 1. Características demográficas, antropométricas e clínicas associadas com osteoporose/fratura e outras co-morbidades na amostra estudada

\begin{tabular}{|c|c|c|}
\hline & $\begin{array}{c}\text { Feminina } \\
(n=462)\end{array}$ & $\begin{array}{c}\text { Masculina } \\
(n=307)\end{array}$ \\
\hline Idade (anos) & $73,5 \pm 5,4$ & $72,7 \pm 5,0$ \\
\hline Peso $(\mathrm{kg})$ & $65,4 \pm 13,4$ & $70,7 \pm 12,3$ \\
\hline Altura $(\mathrm{cm})$ & $150,5 \pm 6,1$ & $162,7 \pm 7,6$ \\
\hline Índice de Massa Corpórea $\left(\mathrm{kg} / \mathrm{m}^{2}\right)$ & $28,8 \pm 5,4$ & $26,6 \pm 3,9$ \\
\hline Cor branca, n (\%) & $304(65,8)$ & $212(69,1)$ \\
\hline Idade da menopausa, anos & $46,1 \pm 10,9$ & NA \\
\hline Etilismo atual, n (\%) & $2(0,43)$ & $32(10,4)$ \\
\hline Tabagismo atual, $\mathrm{n}(\%)$ & $46(10,0)$ & $47(15,3)$ \\
\hline Uso de glicocorticóide, n (\%) & $8(1,7)$ & $3(1,0 \%)$ \\
\hline Fratura clínica por fragilidade, n (\%) & $74(16,0)$ & $18(5,9)$ \\
\hline História familiar de fratura de quadril, n (\%) & $42(9,9)^{*}$ & $18(6,6)^{\star *}$ \\
\hline Caidor crônico, n (\%) & $77(16,6)$ & $28(9,1)$ \\
\hline Ingestão de cálcio (leite e derivados), mg/d & $445 \pm 311$ & $409 \pm 320$ \\
\hline Atividade física alta, n (\%) & $104(22,5)$ & $62(20,2)$ \\
\hline Dorsalgia, n (\%) & $253(54,8)$ & $91(29,6 \%)$ \\
\hline Hipertensão Arterial Sistêmica, n (\%) & $339(73,4)$ & $166(54,1)$ \\
\hline Diabetes Mellitus, n (\%) & $114(24,8)$ & $49(16,0 \%)$ \\
\hline
\end{tabular}

Dados expressos em média \pm desvio-padrão ou porcentagem

NA: não se aplica.

${ }^{*} n=423,{ }^{* *} n=273$ 
A média dos valores de PTH nas mulheres foi de $42,3 \pm 22,4 \mathrm{pg} / \mathrm{ml}$, e nos homens foi de $37,8 \pm 18,1 \mathrm{pg} / \mathrm{ml}$. Os valores médios de vitamina $\mathrm{D}$ foram $18,2 \pm 9,2 \mathrm{ng} / \mathrm{dl}$ nas mulheres e 21,3 $\pm 9,6 \mathrm{ng} / \mathrm{dl}$ nos homens. Os demais parâmetros laboratoriais das populações estão mostrados na tabela 2.

Os resultados da DMO por região nas mulheres foram: $0,839 \pm 0,176 \mathrm{~g} / \mathrm{cm}^{2}$ na coluna lombar, $0,660 \pm 0,125 \mathrm{~g} / \mathrm{cm}^{2}$ no colo femoral; e $0,789 \pm 0,137 \mathrm{~g} / \mathrm{cm}^{2}$ no fêmur total (Tabela 2).

Os valores da DMO por região nos homens foram: 1,009 $\pm 0,196 \mathrm{~g} / \mathrm{cm}^{2}$ na coluna lombar; $0,754 \pm 0,144 \mathrm{~g} / \mathrm{cm}^{2}$ no colo femoral e $0,930 \pm 0,150 \mathrm{~g} / \mathrm{cm}^{2}$ no fêmur total (Tabela 2). 
Tabela 2. Dados laboratoriais, densidade mineral óssea (DMO) nas regiões de coluna lombar, colo femoral e fêmur total na amostra da amostra

\begin{tabular}{lcc}
\hline & $\begin{array}{c}\text { Feminina } \\
(\mathbf{n}=\mathbf{4 6 2})\end{array}$ & $\begin{array}{c}\text { Masculina } \\
(\mathbf{n}=\mathbf{3 0 7})\end{array}$ \\
\hline Cálcio sérico, mg/dL & $9,4 \pm 0,4$ & $9,3 \pm 0,5$ \\
Fósforo, mg/dL & $3,5 \pm 0,5$ & $3,1 \pm 0,5$ \\
Fosfatase alcalina, U/L & $192,4 \pm 60,9$ & $171,0 \pm 64,5$ \\
Creatinina, mg/dL & $0,95 \pm 0,2$ & $1,20 \pm 0,3$ \\
eTFG, mL/min & $56,6 \pm 18,3$ & $59,4 \pm 17,9$ \\
Glicemia, mg/dL & $120,2 \pm 52,5$ & $113,5 \pm 32,6$ \\
PTHi, pg/mL & $42,3 \pm 22,4$ & $37,8 \pm 18,1$ \\
25OHD, ng/mL & $18,2 \pm 9,2$ & $21,3 \pm 9,6$ \\
L1-L4 DMO, g/cm ${ }^{2}$ & $0,834 \pm 0,176$ & $1,009 \pm 0,196$ \\
L1-L4 T-score & $-2,3 \pm 1,6$ & $-1,0 \pm 1,8$ \\
Colo femoral DMO, g/cm ${ }^{2}$ & $0,660 \pm 0,125$ & $0,754 \pm 0,144$ \\
Colo femoral T-score & $-1,8 \pm 1,0$ & $-1,5 \pm 1,0$ \\
Fêmur total DMO, g/cm ${ }^{2}$ & $0,789 \pm 0,137$ & $0,930 \pm 0,150$ \\
Fêmur total T-score & $-1,4 \pm 1,1$ & $-0,9 \pm 0,9$ \\
\hline
\end{tabular}

Dados expressos em média \pm desvio-padrão.

eTFG = estimativa da taxa de filtração glomerular, PTHi = paratormônio intacto $25 \mathrm{OHD}=25$ hidroxivitamina $\mathrm{D}$ 


\subsection{Avaliação das fraturas vertebrais}

Encontrou-se uma prevalência de fratura vertebral de 18,5\% em toda amostra populacional. A freqüência deste evento foi de 16,7\% (77 mulheres) na população feminina e $21,2 \%$ (65 homens) na população masculina $(P=0,156)$. A distribuição das fraturas vertebrais for faixa etária em toda a amostra e por gênero é demonstrada na Tabela 3.

Tabela 3. Prevalência ( $95 \%$ intervalo de confiança) das Fraturas vertebrais por faixa etária e por gênero

\begin{tabular}{lccc}
\hline Idade & Toda & Feminina & Masculina \\
\hline $60-69$ a & $12,7(8,4-17,0)$ & $4,7(1,0-8,4)$ & $22,8(14,6-31)$ \\
$70-79$ a & $18,5(14,9-22,1)$ & $16,8(12,4-21,2$ & $21,1(15,1-27,2)$ \\
80 a ou mais & $33,7(24,0-43,4)$ & $42,6(30,2-55,0)$ & $16,1(3,2-29,1)$ \\
Todas as idades & $18,5(15,8-21,2)$ & $16,7(13,3-20,1)$ & $21,2(16,6-25,7)$ \\
\hline
\end{tabular}

Dados expressos em porcentagem e intervalo de confiança. 


\subsection{Avaliação dos fatores de risco para fraturas vertebrais por população}

\subsubsection{População feminina}

As mulheres do grupo Fratura Vertebral apresentavam maior idade $(77,0 \pm 5,8$ vs. $72,8 \pm 4,9$ anos, $p<0,001)$ e com menor estatura $(148,3 \pm 6,0$ vs. $151,0 \pm 6,0 p<0,001)$ quando comparadas ao grupo Sem Fratura Vertebral. Não houve diferença entre os dois grupos com relação ao peso, IMC e cor branca (Tabela 4).

A prática de atividade física foi diferente nos dois grupos. Mulheres do grupo Fratura Vertebral foram menos frequentemente classificadas como praticantes de atividade física alta $(10,4$ vs. $24,9 \%, p=0,005)$. Os dois grupos eram semelhantes no que diz respeito aos demais parâmetros clínicos avaliados (Tabela 4). 
Tabela 4. Dados demográficos, antropométricos e clínicos da amostra feminina distribuídos no grupo Fratura Vertebral e Sem Fratura Vertebral

\begin{tabular}{|c|c|c|c|}
\hline & $\begin{array}{c}\text { Fratura } \\
\text { Vertebral } \\
(n=77)\end{array}$ & $\begin{array}{c}\text { Sem } \\
\text { Fratura } \\
\text { Vertebral } \\
(n=385)\end{array}$ & $\mathbf{P}$ \\
\hline Idade (anos) & $77,0 \pm 5,8$ & $72,8 \pm 4,9$ & $<0,001$ \\
\hline Peso (kg) & $63,9 \pm 14,3$ & $65,7 \pm 13,3$ & 0,324 \\
\hline Altura $(\mathrm{cm})$ & $148,3 \pm 6,0$ & $151,0 \pm 6,0$ & $<0,001$ \\
\hline Índice de Massa Corpórea $\left(\mathrm{kg} / \mathrm{m}^{2}\right)$ & $29,0 \pm 5,9$ & $28,7 \pm 5,3$ & 0,686 \\
\hline Cor branca, n (\%) & $54(70,1)$ & $250(64,9)$ & 0,380 \\
\hline Tabagismo atual, $\mathrm{n}(\%)$ & $4(5,2)$ & $42(10,9)$ & 0,126 \\
\hline Uso de glicocorticóide, n (\%) & $3(3,9)$ & $5(1,3)$ & 0,111 \\
\hline Fratura por fragilidade, $\mathrm{n}(\%)$ & $16(20,8)$ & $54(14,0)$ & 0,131 \\
\hline História familiar de fratura de quadril, n (\%) & $3(4,4)^{*}$ & $39(11,02)^{\star *}$ & 0,090 \\
\hline Caidor crônico, n (\%) & $14(18,2)$ & $63(16,4)$ & 0,696 \\
\hline Ingestão de cálcio (leite e derivados), mg/dia & $425 \pm 326$ & $449 \pm 309$ & 0,541 \\
\hline Atividade física alta, $\mathrm{n}(\%)$ & $8(10,4)$ & $96(24,9)$ & 0,005 \\
\hline Dorsalgia, n (\%) & $44(57,1)$ & $209(54,3)$ & 0,646 \\
\hline Hipertensão Arterial Sistêmica, n (\%) & $50(64,9)$ & $289(75,1)$ & 0,066 \\
\hline Diabetes Mellitus, n (\%) & $17(22,1)$ & $97(25,2)$ & 0,563 \\
\hline
\end{tabular}

Dados expressos em média \pm desvio-padrão ou porcentagem ${ }^{*} n=69,{ }^{* *} n=354$ 
O grupo Fratura Vertebral apresentava menor eTFG $(52,5 \pm 18,5$ vs. $57,4 \pm 18,1 \mathrm{~mL} / \mathrm{min}, \mathrm{p}=0,030)$, maiores valores séricos de PTHi $(47,1 \pm 26,0$ vs. $41,3 \pm 21,5 \mathrm{pg} / \mathrm{mL}, \mathrm{p}=0,037)$ e menores valores séricos de $250 \mathrm{HD}(15,5$ $\pm 7,3$ vs. $18,7 \pm 9,5 \mathrm{ng} / \mathrm{mL}, \mathrm{p}=0,005)$. Cálcio, fósforo, fosfatase alcalina, creatinina e glicemia apresentaram valores semelhantes entre os dois grupos (Tabela 5).

Em relação aos dados densitométricos o grupo Fratura Vertebral apresentou menor DMO de colo femoral $(0,600 \pm 0,123$ vs. 0,672 \pm $\left.0,122 \mathrm{~g} / \mathrm{cm}^{2} \mathrm{p}<0,001\right)$ e do fêmur total $\left(0,716 \pm 0,142\right.$ vs. $0,804 \pm 0,132 \mathrm{~g} / \mathrm{cm}^{2}$, $p<0,001)$ comparado com o grupo Sem Fratura Vertebral. Valores da DMO de L1-L4 foram semelhantes nos dois grupos (Tabela 5). 
Tabela 5. Dados laboratoriais e da densidade mineral óssea (DMO) nas regiões de coluna lombar, colo femoral, fêmur total, da amostra feminina distribuídos nos grupos Fratura Vertebral e Sem Fratura Vertebral

\begin{tabular}{lccc}
\hline & $\begin{array}{c}\text { Fratura } \\
\text { Vertebral } \\
\mathbf{( n = 7 7 )}\end{array}$ & $\begin{array}{c}\text { Sem Fratura } \\
\text { Vertebral } \\
(\mathbf{n}=\mathbf{3 8 5})\end{array}$ & $\mathbf{P}$ \\
\hline Cálcio sérico, mg/dL & $9,4 \pm 0,4$ & $9,4 \pm 0,5$ & 0,500 \\
Fósforo, mg/dL & $3,6 \pm 0,5$ & $3,5 \pm 0,5$ & 0,242 \\
Fosfatase alcalina, U/L & $200,8 \pm 85,7$ & $190,7 \pm 54,7$ & 0,184 \\
Creatinina, mg/dL & $0,96 \pm 0,2$ & $0,95 \pm 0,2$ & 0,598 \\
eTFG, mL/min & $52,5 \pm 18,5$ & $57,4 \pm 18,1$ & $\mathbf{0 , 0 3 0}$ \\
Glicemia, mg/dL & $113,6 \pm 26,6$ & $121,6 \pm 56,2$ & 0,225 \\
PTHi, pg/mL & $47,1 \pm 26,0$ & $41,3 \pm 21,5$ & $\mathbf{0 , 0 3 7}$ \\
25OHD, ng/mL & $15,5 \pm 7,3$ & $18,7 \pm 9,5$ & $\mathbf{0 , 0 0 5}$ \\
L1-L4 DMO, g/cm ${ }^{2}$ & $0,799 \pm 0,204$ & $0,840 \pm 0,169$ & 0,057 \\
L1-L4 T-score & $-2,5 \pm 1,8$ & $-2,2 \pm 1,5$ & 0,092 \\
Colo femoral DMO, g/cm & $0,600 \pm 0,123$ & $0,672 \pm 0,122$ & $<\mathbf{0 , 0 0 1}$ \\
Colo femoral T-score & $-2,3 \pm 1,0$ & $-1,7 \pm 1,0$ & $<\mathbf{0 , 0 0 1}$ \\
Fêmur total DMO, g/cm & $0,716 \pm 0,142$ & $0,804 \pm 0,132$ & $<\mathbf{0 , 0 0 1}$ \\
Fêmur total, T-score & $-1,9 \pm 1,1$ & $-1,2 \pm 1,01$ & $<\mathbf{0 , 0 0 1}$ \\
\hline
\end{tabular}

Dados expressos em média \pm desvio-padrão,

eTFG = estimativa da taxa de filtração glomerular, PTHi = paratormônio intacto, $25 \mathrm{OHD}=25$ hidroxivitamina $\mathrm{D}$ 
$\mathrm{Na}$ análise de regressão logística da população feminina foram incluídas as variáveis com significância $(p<0,05)$ na análise univariada: idade, altura, atividade física alta, eTFG, PTHi, $250 H D$ e os valores do T-score do colo femoral ou do fêmur total. Modelos de regressão foram criados com o T-score destes dois sítios (colo de fêmur, fêmur total) individualmente. Os dados mostraram-se ajustados para altura, atividade física alta, eTFG, PTHi e 25OHD, tendo a idade e os valores de T-score do colo femoral ou do fêmur total permanecidos como fatores independentes para a presença de fratura vertebral moderada ou grave (Tabela 6).

Tabela 6. Análise de regressão logística para a presença de fraturas vertebrais moderada/grave na população feminina

\begin{tabular}{lccc}
\hline & OR & Intervalo de Confiança 95\% & P \\
\hline Idade & 1,12 & $(1,06-1,18)$ & $<0,001$ \\
Colo femoral T-score & 0,64 & $(0,46-0,88)$ & 0,006 \\
\hline ou & OR & Intervalo de Confiança 95\% & P \\
\hline & 1,11 & $(1,06-1,17)$ & $<0,001$ \\
\hline Idade & 0,61 & $(0,45-0,84)$ & 0,002 \\
\hline
\end{tabular}

OR ajustado por altura, atividade física alta, eTFG, PTHi e 25OHD 


\subsubsection{População Masculina}

Não houve diferença significativa entre os grupos Fratura Vertebral e Sem Fratura Vertebral relacionada aos dados demográficos e antropométricos nos homens. A proporção de caidores crônicos foi maior nos homens do grupo Fratura Vertebral do que nos homens do grupo Sem Fratura Vertebral $(15,4$ vs. $7,4 \%, p=0,048)$. Demais parâmetros clínicos não apresentaram diferença entre esses dois grupos nesta população (Tabela 7).

Tabela 7. Dados demográficos, antropométricos e clínicos da amostra masculina distribuídos nos grupos Fratura Vertebral e Sem Fratura Vertebral

\begin{tabular}{lccc}
\hline & $\begin{array}{c}\text { Fratura } \\
\text { Vertebral } \\
(\mathbf{n = 6 5 )}\end{array}$ & $\begin{array}{c}\text { Sem } \\
\text { Fratura } \\
\text { Vertebral } \\
(\mathbf{n = 2 4 2})\end{array}$ & $\mathbf{P}$ \\
\hline Idade (anos) & $72,8 \pm 5,0$ & $72,7 \pm 5,0$ & 0,979 \\
Peso (kg) & $72,8 \pm 13,5$ & $70,1 \pm 12,0$ & 0,116 \\
Altura (cm) & $163,4 \pm 8,2$ & $162,5 \pm 7,4$ & 0,418 \\
IMC (kg/m $\left.{ }^{2}\right)$ & $27,2 \pm 4,5$ & $26,5 \pm 3,7$ & 0,162 \\
Brancos, n(\%) & $44(67,7)$ & $168(69,4)$ & 0,789 \\
História familiar de fratura de quadril, n (\%) & $6(10,9)^{*}$ & $12(5,5)^{\star *}$ & 0,149 \\
Caidor crônico, $\mathrm{n}(\%)$ & $10(15,4)$ & $18(7,4)$ & $\mathbf{0 , 0 4 8}$ \\
Ingestão de leite e derivados, mg/d & $408 \pm 333$ & $410 \pm 317$ & 0,965 \\
Atividade física alta, n(\%) & $10(15,4)$ & $52(21,5)$ & 0,277 \\
Dorsalgia, n(\%) & $18(27,7)$ & $73(30,2)$ & 0,698 \\
HAS, n(\%) & $36(55,4)$ & $130(53,7)$ & 0,811 \\
Diabetes Mellitus, n(\%) & $8(12,3)$ & $41(16,9)$ & 0,365 \\
\hline
\end{tabular}

Dados expressos em média \pm desvio-padrão ou porcentagem ${ }^{*} n=22,{ }^{* *} n=308$ 
Não houve diferenças entre os parâmetros laboratoriais avaliados nos dois grupos Fratura Vertebral e Sem Fratura Vertebral entre os homens (Tabela 8).

Da mesma forma que nas mulheres, as DMOs do colo femoral e do fêmur total foram menores nos homens do grupo Fratura Vertebral do que nos homens do grupo Sem Fratura Vertebral $(0,719 \pm 0,133$ vs $0,764 \pm 0,146, p<0,001 ; 0,895 \pm 0,133$ vs. $0,940 \pm 0,154, p<0,001$ respectivamente). Demais parâmetros, DMO de L1-L4 não diferiram entre os homens dos dois grupos (Tabela 8). 
Tabela 8. Dados laboratoriais e da densidade mineral óssea (DMO) nas regiões de coluna lombar, colo femoral, fêmur total, da amostra masculina distribuída nos dois grupos Fratura Vertebral e Sem Fratura Vertebral

\begin{tabular}{lccc}
\hline & $\begin{array}{c}\text { Fratura } \\
\text { Vertebral } \\
(\mathbf{n}=\mathbf{6 5})\end{array}$ & $\begin{array}{c}\text { Sem Fratura } \\
\text { Vertebral } \\
\mathbf{( n = 2 4 2}\end{array}$ & $\mathbf{P}$ \\
\hline Cálcio sérico, mg/dL & $9,2 \pm 0,4$ & $9,3 \pm 0,5$ & 0,171 \\
Fósforo, mg/dL & $3,2 \pm 0,6$ & $3,1 \pm 0,5$ & 0,262 \\
Fosfatase alcalina, U/L & $167,5 \pm 48,9$ & $171,9 \pm 68,1$ & 0,628 \\
Creatinina, mg/dL & $1,14 \pm 0,3$ & $1,17 \pm 0,2$ & 0,569 \\
TFGe, mL/min & $62,4 \pm 18,8$ & $58,5 \pm 17,6$ & 0,121 \\
Glicemia, mg/dL & $112,8 \pm 41,1$ & $113,7 \pm 30,1$ & 0,840 \\
PTHi, pg/mL & $37,5 \pm 16,8$ & $37,8 \pm 18,5$ & 0,914 \\
25OHD, ng/mL & $22,3 \pm 11,3$ & $21 \pm 9,1$ & 0,318 \\
L1-L4 DMO, g/cm ${ }^{2}$ & $0,986 \pm 0,181$ & $1,015 \pm 0,200$ & 0,286 \\
L1-L4 T-score & $-1,2 \pm 1,7$ & $-0,9 \pm 1,8$ & 0,318 \\
Colo femoral DMO, g/cm & $0,719 \pm 0,133$ & $0,764 \pm 0,146$ & $\mathbf{0 , 0 2 6}$ \\
Colo femoral T-score & $-1,8 \pm 1,0$ & $-1,4 \pm 1,1$ & $\mathbf{0 , 0 3 8}$ \\
Fêmur total DMO, g/cm & $-1,1 \pm 0,9$ & $-0,8 \pm 1,0$ & 0,051 \\
Fêmur total T-score & $0,895 \pm 0,133$ & $0,940 \pm 0,154$ & $\mathbf{0 , 0 3 0}$ \\
\hline Dados & & & \\
\hline
\end{tabular}

Dados expressos em média \pm desvio-padrão,

eTFG = estimativa da taxa de filtração glomerular, PTHi = paratormônio intacto, $25 \mathrm{OHD}=25$ hidroxivitamina $\mathrm{D}$ 
A análise de regressão logística da população masculina foi construída com modelos incluindo etilismo atual e valores da DMO de cada sítio (colo femoral e fêmur total) individualmente. Apenas as DMOs de cada sítio permaneceram nos modelos como fatores significantes para a presença de fraturas vertebrais (Tabela 9).

Tabela 9. Análise de regressão logística para a presença de fraturas vertebrais moderada/grave na amostra masculina

\begin{tabular}{lccc}
\hline & OR & Intervalo de Confiança 95\% & P \\
\hline Colo femoral T-score & 0,72 & $(0,53-0,96)$ & 0,025 \\
Caidor & 2,54 & $1,1-5,9$ & 0,031 \\
\hline
\end{tabular}


Este é o primeiro estudo epidemiológico realizado no Brasil desenhado especificamente para avaliar fraturas vertebrais, analisando concomitantemente a densidade mineral óssea, parâmetros bioquímicos do metabolismo ósseo e fatores de risco em indivíduos idosos, com 65 anos ou mais. A população estudada pode ser considerada uma amostra representativa da população de idosos do Brasil por possuir as mesmas características de distribuição por faixa etária, gênero e classe social como mostrado pelos dados do censo de 2000 (IBGE, 2002).

A grande vantagem do presente estudo é o uso da técnica semiquantitativa (SQ) de Genant para o diagnóstico de fratura vertebral, uma vez que esta técnica é mais objetiva e reproduzível que outros métodos com melhor concordância interobservador, e seu sistema de graduação constitui uma ferramenta útil para uso em estudos epidemiológicos e ensaios clínicos de osteoporose (Genant et al., 1996; Ferrar et al., 2005). Devido à simplicidade do sistema de graduação deste método, ele também pode ser facilmente usado na prática clínica por não ser necessário o conhecimento da altura vertebral normal da população brasileira para o diagnóstico de fratura.

Encontrou-se uma prevalência de fraturas vertebrais moderadas/graves de $18,5 \%$ para fraturas vertebrais, sendo $16,7 \%$ nas mulheres e $21,2 \%$ e nos homens. 
Em relação às fraturas vertebrais, a freqüência encontrada na nossa amostra entre as mulheres foi superior à encontrada na América Latina $(14,7 \%)$ e no Brasil $(14,2 \%)$ segundo o estudo LAVOS (Clark et al., 2009). Este resultado pode ser devido à população analisada no nosso estudo ser mais idosa que a população estudada no LAVOS $(73,5 \pm 5,4$ vs. $68,36 \pm$ 10,96 anos, $p<0,001)$. De fato, comparando-se a distribuição de fraturas por faixa etária na população feminina, a maior prevalência de fratura vertebral em nosso estudo pode ser devido à elevada frequência de fraturas na faixa etária de 80 anos ou mais. Outra possível explicação pode ser o uso de outro método pelo estudo LAVOS para definir fratura. No estudo LAVOS, os autores definiram fraturas vertebrais como uma redução de $3 \mathrm{DP}$ da média normal para a altura vertebral (Clark et al, 2009).

Estudos europeus (O'Neill et al.,1996) mostraram uma prevalência de fraturas vertebrais de $12 \%$ para mulheres e $12,2 \%$ para homens, porém a faixa etária analisada era mais jovem (50-84 anos) que no nosso estudo (65-94 anos).

Nós confirmamos observações prévias que apontam a idade como importante fator preditor de fraturas vertebrais em mulheres (El Maghraoui et al, 2008). O aumento da prevalência com o avançar da idade concorda com os dados do estudo LAVOS. Neste estudo a prevalência de 6,9\% nas mulheres de faixa etária de 50-59 anos aumentou para $27,8 \%$ nas mulheres maiores de 80 anos (Clark et al., 2009).

O achado do quadril como o melhor parâmetro da densitometria para predizer fraturas vertebrais tanto em mulheres como em homens, em relação à 
coluna lombar, pode ser explicado pela frequência de fatores de confusão que aumentam a massa óssea na coluna lombar de indivíduos idosos, tais como osteófitos, calcificação vascular e a própria fratura vertebral (Reid et al., 1991; Masud et al., 1993; Muraki et al., 2004; Schneider et al., 2006).

A associação de fraturas vertebrais com o fato de o paciente ser um caidor é interessante, é isto é bem demonstrado em relação a fraturas por fragilidade no quadril (Cumming et al., 1994; Greenspan et al., 1994; Sherrington et al.,1998). Estudos observacionais e caso-controle implicam que a melhora do equilíbrio e da força muscular podem reduzir o risco de quedas e consequentemente o risco de fraturas de quadril (Hourigan et al., 2008; Karlsson et al.; 2008). Se a melhora do equilíbrio e da força muscular reduz outras fraturas por fragilidade incluindo fraturas vertebrais deve ser melhor avaliado em estudos posteriores.

Finalmente, é importante enfatizar que fraturas vertebrais prévias estão associadas à piora da qualidade de vida (Pasco et al, 2009), limitações funcionais (O'Neill et al, 2004) e aumento da morbidade e da mortalidade (Hasserius et al, 2005). Estudos mostram que a taxa de mortalidade após cinco anos do diagnóstico de uma fratura vertebral pode aumentar em cerca de vinte por cento em relação ao esperado (Ensured et al, 2000). Mulheres pós-menopausadas com fraturas vertebrais graves tem um alto risco para fraturas subseqüentes vertebrais e não vertebrais (Ismail et al, 2001). Realmente, a gravidade das fraturas vertebrais mostrou ser melhor do que a DMO para predizer futuras fraturas não vertebrais (Delmas et al, 2003). Da mesma forma, fraturas prévias não vertebrais representam aumento de risco para outras fraturas (Klotzbuecher et al, 2000). 
Em conclusão, nossos resultados mostraram que as fraturas vertebrais são comuns em idosos da comunidade e suportam a nova recomendação da ISCD para investigação de fraturas vertebrais em indivíduos maiores de 65 anos, sobretudo se estes apresentarem baixa DMO (Schousboe et al, 2007). O uso da identificação de fraturas vertebrais neste estudo pela técnica semiquantitativa é importante na prática clínica, uma vez que esta técnica pode ser facilmente utilizada por clínicos treinados, não necessitando do conhecimento da altura vertebral normal para a população estudada.

Diante disso, nossos resultados abrem caminho para que e médicos e autoridades no Brasil, possam reconhecer a importância de programar medidas apropriadas para prevenção e tratamento de fraturas vertebrais, no intuito de reduzir esta ocorrência de na população. 
Fraturas vertebrais são frequentes em idosos da comunidade, com prevalência de $16,7 \%$ nas mulheres e $21,2 \%$ nos homens.

Fraturas vertebrais moderadas e graves nesta população são associadas com:

- Baixo T-score do colo femural em homens e mulheres;

- Idade em mulheres;

- Condição de caidor crônico em homens. 


\section{REFERÊNCIAS}


Anonymous WHO Study Group. Assessment of fracture risk and its application to screening for postmenopausal osteoporosis. Report of a WHO Study Group. World Health Organ Tech Rep Ser. 1994; 843: 1-129.

Black DM, Reiss TF, Nevitt MC, Cauley J, Karpf D at al Design of the Fracture Intervention Trial. Osteoporos Int. 1993;3:S29-39

Cauley JA, Hochberg MC, Lui L, Palermo L, Ensrud KE, Hillier TA, Nevitt MC, Cummings SR. Long-term risk of incident vertebral fractures. JAMA. 2007;298:2761-7

Clark P, Cons-Molina F, Deleze M, Ragi S, Haddock L, Zanchetta JR et al. The prevalence of radiographic vertebral fractures in Latin American countries: the Latin American Vertebral Osteoporosis Study (LAVOS). Osteoporos Int. 2009; 20:275-82

Cockcroft DW, Gault MH. Prediction of creatinine clearance from serum creatinine. Nephron. 1976; 16:31-41

Consensus Development Conference: diagnosis, prophylaxis and treatment of osteooporis. Am J Med. 1993; 94:646-50 
Cooper C, Atkinson EJ, O'Fallon WM, Melton LJ. Incidence of clinically diagnosed vertebral fractures: A population-based study in Rochester, Minesota, 1985-1989. J Bone Miner Res. 1992;7:221-7

Cumming RG, Klineberg RJ. Fall frequency and characteristics and the risk of hip fractures. J Am Geriatr Soc. 1994;42:774-8

Delmas PD, Genant HK, Crans GG, Stock JL, Wong M, Siris E, Adachi JD. Severity of prevalent vertebral fractures and the risk of subsequent vertebral and nonvertebral fractures: results from the MORE trial. Bone. 2003;33:522-32

Delmas PD, van de Langerijt L, Watts NB, Eastell R, Genant H, Grauer A et al. Underdiagnosis of Vertebral Fractures is a Worldwide Problem: The IMPACT Study Group. J Bone Miner Res. 2005;20:557-63

El Maghraoui A, Mounach A, Gassim S, Ghazi M. Vertebral fracture assessment in healthy men: prevalence and risk factors. Bone. 2008;43:544-8

Ensrud KE, Thompson DE, Cauley JA, Nevitt MC, Kado DM, Hochberg MC et al. Prevalent vertebral deformities predict mortality and hospitalization in older women with low bone mass. Fracture Intervention Trial Research Group. J Am Geriatr Soc. 2000;48:241-9 
Ferrar L, Jiang G, Adams J, Eastell R. Identification of vertebral fractures:un update. Osteoporis Int. 2005;16:717-28

Ferrar L, Jiang G, Cawthon PM, San Valentin R, Fullman R, Lambert L et al. Identification of vertebral fracture and non-osteoporotic short vertebral height in men. The MrOS study. J Bone Miner Res. 2007;1434-41

Fitti JE, Kovar M. The Supplement on Aging to the 1984 National Health Interview Survey. Vital Health Stat. 1987;1:1-115

Fuchs SC, Guimarães SM, Sortica C, Wainberg F, Dias KO, Ughini M et al. Reliability of race assessment based on the race of the ascendants: a crosssectional study. BMC Public Health. 2002;2:1-5

Genant HK, WuCY, van Kuijk C, Nevitt MC. Vertebral fracture assessment using a semiquantitative technique. J Bone Miner Res. 1993;8:1137-48

Genant HK, Jergas M, Palermo L, Nevitt M, Valentin RS, Black D, Cummings SR. Comparison of semiquantitative visual and quantitative morphometric assessment of prevalent and incident vertebral fractures in osteoporosis. The Study of Osteoporotic Fractures Research Group. J Bone Miner Res. 1996;11:984-96. 
Greenspan SL, Myers ER, Maitland LA, Resnick NM, Hayes WC. Fall severity and bone mineral density as risk factors for hip fracture in ambulatory elderly. JAMA. 1994;271:128-133.

Harris ST, Watts NB, Genant HK, McKeever CD, Hangartner T, Keller M et al. Effects of risedronate treatment on vertebral and nonvertebral fractures in women with postmenopausal osteoporosis: a randomized controlled trial. Vertebral Efficacy With Risedronate Therapy (VERT) Study Group. JAMA. 1999;282:1344-52.

Hasserius R, Karlsson MK, Jonsson B, Redlund-Johnell I, Johnell O. Longterm morbidity and mortality after a clinically diagnosed vertebral fracture in the elderly--a 12- and 22-year follow-up of 257 patients. Calcif Tissue Int. $2005 ; 76: 235-42$

Hourigan SR, Nitz JC, Brauer SG, O'Neill S, Wong J, Richardson CA. Positive effects of exercise on falls and fracture risk in osteopenic women. Osteoporos Int. 2008;19:1077-86

Instituto Brasileiro de Geografia e Estatística - IBGE. Perfil dos Idosos Responsáveis pelos Domicílios no Brasil 2000. Estudos \& Pesquisas. Informação Sociodemográfica e Socioeconômica 9. Rio de Janeiro: IBGE, 2002. 
Instituto Brasileiro de Geografia e Estatística 2006. Censo 2000: Brazil http://www.ibge.gov.br/censo; acessed 20 March 2007

Ismail AA, Cockerill W, Cooper C, Finn JD, Abendroth K, Parisi G et al. Prevalent vertebral deformity predicts incident hip though not distal forearm fracture: results from the European Prospective Osteoporosis Study. Osteoporos Int. 2001;12:85-90

Karlsson MK, Nordqvist A, Karlsson C. Physical activity, muscle function, falls and fractures. Food Nutr Res. 2008;52.

Lentle BC, Brown JP, Khan A, Leslie WD, Levesque J, Lyons DJ et al. Recognizing and reporting vertebral fractures: reducing the risk of future osteoporotic fractures. Can Assoc Radiol J. 2007;58:27-36

Masud T, Langley S, Wiltshire P, Doyle DV, Spector TD. Effect of spinal osteophytosis on bone mineral density measurements in vertebral osteoporosis. BMJ. 1993;307:172-3

Melton I, Joseph L, Kan SH, Frye MA, Wahner HW, O'Fallon WM, Riggs BL. Epidemiology of vertebral fractures in women. Am $\mathrm{J}$ Epidemiol. 1989;129:1000-10. 
Muraki S, Yamamoto S, Ishibashi H, Horiuchi T, Hosoi T, Orimo H, Nakamura K. Impact of degenerative spinal diseases on bone mineral density of the lumbar spine in elderly women. Osteoporos Int. 2004;15:724-8

O'Neill TW, Cooper C, Cannata JB, Diaz Lopez JB, Hoszowski K, Johnell O et al. Reproducibility of a questionnaire on risk factors for osteoporosis in a multicentre prevalence survey: the European Vertebral Osteoporosis Study. Int J Epidemiol. 1994;23:559-65

O'Neill TW, Felsenberg D, Varlow J, Cooper C, Kanis JA, Silman AJ. The prevalence of vertebral deformity in european men and women: the European Vertebral Osteoporosis Study. J Bone Miner Res. 1996;11:1010-8

Pinheiro MM, Ciconelli RM, Martini LA, Ferraz MB. Clinical risk factors for osteoporotic fractures in Brazilian women and men: the Brazilian Osteoporosis Study (BRAZOS). Osteoporos Int. 2009;20:399-408

Reginster JY, Seeman E., De Vernejoul MC, Adami S, Compston J, Phenekos C et al. Strontium Ranelate Reduces the Risk of Nonvertebral Fractures in Postmenopausal Women with Osteoporosis: Treatment of Peripheral Osteoporosis (TROPOS) Study. J Clin Endocrinol Meta. 2005;90:2816-22 
Reid IR, Evans MC, Ames R, Wattie DJ. The influence of osteophytes and aortic calcification on spinal mineral density in postmenopausal women. $J$ Clin Endocrinol Metab. 1991;72:1372-4

Samelson EJ, Hannan MT, Zhang Y, Genant HK, Felson DT, Kiel DP. Incidence and risk factors for vertebral fracture in women and men: 25-year follow-up results from the population-based Framingham study. $\mathrm{J}$ Bone Miner Res. 2006;21:1207-14

Schneider DL, Bettencourt R, Barrett-Connor E. Clinical utility of spine bone density in elderly women. J Clin Densitom. 2006;9: 255-260

Schousboe JT, Vokes T, Broy SB, Ferrar L, McKiernan F, Roux C et al. Vertebral Fracture Assessment: the 2007 ISCD Official Positions. J Clin Densitom. 2008;11:92-108.

Schwartz AV, Villa ML, Prill M, Kelsey JA, Galinus JA, Delay RR et al. Falls in older Mexican-American womem. J Am Geriatr Soc. 1999;47:1371-8

Seeley DG, Browner WS, Nevitt MC, Genant HK, Scott JC, Cummings SR. Which fractures are associated with low appendicular bone mass in elderly women? The Study of Osteoporotic Fractures Research Group. Ann Intern Med. 1991;115:837-42 
Shepherd JA, Lu Y, Wilson K, Fuerst T, Genant H, Hangartner TN et al. Cross-calibration and Minimum Precision Standards for Dual-Energy X-ray Absorptiometry: The 2005 ISCD Official Positions. J Clin Densitom. $2006 ; 9: 31-6$

Sherrington C, Lord SR. Increased prevalence of fall risk factors in older people following hip fracture. Gerontology. 1998;44:340-4.

Siris E, Adachi JD, Lu Y, Fuerst T, Crans GG, Wong M, Harper KD, Genant HK. Effects of raloxifene on fracture severity in postmenopausal women with osteoporosis: results from the MORE study. Multiple Outcomes of Raloxifene Evaluation. Osteoporos Int. 2002;13:907-13

United Nations Population Division, World Population Prospects: The 1998 revision; 1998. 
Apêndices 


\section{Prevalence and risk factors of radiographic vertebral fracture in Brazilian community-dwelling elderly}

Jaqueline B. Lopes, Camille F. Danilevicius, Liliam Takayama, Valeria F. Caparbo, Paulo R. Menezes $^{1}$, Marcia Scazufca ${ }^{2}$, Marcia E. Kuroishi ${ }^{3}$, Rosa M. R. Pereira.

JB Lopes, MD; CF Danilevicius, MD; Takayama L, Biologist; VF Caparbo, Biologist; PR Menezes, MD, PhD, M Scazufca, PhD; ME Kuroishi, MD; RMR Pereira, MD, PhD.

Bone Metabolism Laboratory, Rheumatology Division; Department of Preventive Medicine ${ }^{1}$ and Departament of Psychiatry ${ }^{2}$, Radiology Division of Hospital Universitário ${ }^{3}$, Faculdade de Medicina da Universidade de São Paulo, São Paulo, Brazil

\section{Corresponding author:}

Rosa M. R. Pereira

Faculdade de Medicina da USP, Laboratório de Metabolismo Ósseo (LIM17), Disciplina de Reumatologia, FMUSP

Av. Dr. Arnaldo, 455, Sala 3105

São Paulo, SP, 01246-903, Brazil

Telephone: +55-11-3061-7213

Fax: +55-11-3061-7490

E-mail: rosamariarp@yahoo.com 


\section{Mini-abstract}

The prevalence and risk factors of radiographic vertebral fracture were determined among Brazilian community-dwelling elderly. Vertebral fractures were a common condition in this elderly population, and femoral neck T-score was a significant risk factor in both genders. 


\section{Abstract}

Purpose: To estimate the prevalence of radiographic vertebral fracture and investigate factors associated with this condition in Brazilian community-dwelling elderly.

Methods: This cross sectional study included 769 elderly subjects (462 women and 307 men) living in São Paulo, Brazil. Thoracic and lumbar spine radiographs were obtained and vertebral fractures were evaluated using Genant's semi-quantitative method. Bone mineral density (BMD) was measured by DXA and bone biochemical markers were also evaluated. Female and male subjects were analyzed independently, and each gender was divided into 2 groups, based on whether vertebral fractures were present.

Results: The prevalence of vertebral fracture was $16.7 \%(95 \%$ CI $13.3-20.1)$ in women and $21.2 \%(95 \% \mathrm{CI} 16.6-25.7)$ in men. Logistic regression analyses using variables that were significant in the univariate analysis, in female group showed that age $(\mathrm{OR}=1.12,95 \% \mathrm{CI}$ 1.06-1.18; $\mathrm{p}<0.001)$ and femoral neck $\mathrm{T}$-score $(\mathrm{OR}=0.61,95 \% \mathrm{CI} 0.46-0.88 ; \mathrm{p}=0.006)$ were independently factors in predicting vertebral fracture. In the male group, logistic regression analyzes demonstrated that chronic faller condition $(\mathrm{OR}=2.5495 \% \mathrm{CI} 1.1-5.9 ; \mathrm{p}=0.031)$ and femoral neck T-score $(\mathrm{OR}=0.72,95 \% \mathrm{CI} \quad 0.53-0.96 ; \mathrm{p}=0.025)$ were independent parameters in predicting vertebral fractures.

Conclusions: Our results suggest that radiographic vertebral fractures are common in Brazilian community-dwelling elderly and that a low femoral neck T-score was an important risk factor for this condition in both males and females. Age was also significantly correlated with the presence of vertebral fractures in women, and chronic faller was correlated with vertebral fractures in men.

Key terms: vertebral fracture, elderly people, bone mineral density, femur, fall, radiography 


\section{Introduction}

Underdiagnosis of vertebral fracture remains a major problem worldwide [1]. Thus, in studies using radiographic screening of populations, the incidence of all vertebral deformities has been estimated to be three times higher than hip fracture, and only one third of vertebral fractures receive medical attention [2]. Vertebral fractures have important clinical implications for future fracture risk. Studies have demonstrated that prevalent vertebral fracture can predict subsequent vertebral fracture [3] incident hip fracture [4], and increased mortality in following decades [5], particularly if the resulting vertebral deformity is severe [3]. As the world population ages, the public health impact of vertebral fractures will increase, particularly in low and middle income countries, where ageing process has been much faster.

Prevalence of risk factors associated with osteoporosis (OP), including bone mineral density (BMD) and bone laboratory parameters associated with vertebral fractures have not been well characterized in healthy and asymptomatic subjects. Research has been particularly lacking in populations from low and middle income countries, like Brazil $[6,7]$. A recent study analyzed the prevalence of vertebral fractures in Latin American women [7]. Nevertheless, this study was not specifically designed to characterize risk factors, and bone mineral density and bone laboratory parameters were not assessed [7].

The standard method for assessing vertebral fracture is radiographic analysis of the thoracic and lumbar spine region; however, a number of methods have been developed for interpretation of spinal X-rays, including the Genant semi-quantitative method [8]. The Genant semi-quantitative method has been used to diagnose vertebral fractures in physicians guidelines [9] and has been the standard in a number of important recent osteoporosis studies [10-15].

The aim of this study was to determine the prevalence of radiographic vertebral fracture in Brazilian community-dwelling elderly using Genant's semi-quantitative (SQ) method. The study further aimed to determine the association between these fractures and OP risk factors including BMD and bone laboratory parameters. 


\section{Material and Methods}

\section{Subjects}

This was a cross-sectional study nested in a population-based epidemiological study (São Paulo Ageing \& Health Study). The survey was conducted from June 2005 to July 2007 on individuals 65 years old and over living in the community in the Butantã district, located on the western side of the city of São Paulo (subtropical region, latitude 2332'51").

This city supports a population of 10.4 million inhabitants [16] and is divided into 31 administrative boroughs, with territorial and population demarcations. In 2000, the Butantã district contained 377,576 residents, $6.2 \%$ of whom were over 65 years of age. The study was carried out in 66 census sectors (the smallest administrative areas) covering a population of approximately 63,000 residents, representing $17 \%$ of the total Butantã borough population. The selection of areas was based on the Family Health Program teams, but was not limited by them (i.e., they included the entire census areas). This sample was representative of the age, gender and social class demographics of the entire Brazilian elderly population [17].

Only well-functioning elderly were recruited to participate in this study on osteoporosis. All of the individuals were apparently healthy, and showed no evidence of malabsorption, chronic diarrhea, hepatic disease, severe chronic diseases or cancer.

All individuals answered a standardized questionnaire designed to document putative risk factors of osteoporosis and fractures [18-19]. The questionnaire collected information regarding their health and lifestyle, including family history of fragility fracture, previous fragility fracture, history of falls during the last year (two or more falls in 6 months, any fall in the last year resulting in serious injury), physical activity, alcohol use, current tobacco use, glucocorticoid use, presence of back pain, dietary calcium and age at menopause.

Previous fragility fracture was determined in individuals who had experienced a fall from standing height or less after 50 years of age, and occurring at sites characteristic of 
bone fragility (for example, rib, forearm, humerus, and femur). Fractures occurring of the face, skull, ankle, elbow and finger were not considered in this analysis [20]. Individuals who had experienced two or more falls in the last 12 months were defined as chronic fallers [21]. An individual was deemed to be an alcohol user when current intake exceeded three units of alcohol per day. Glucocorticoid use was defined as prednisone treatment of more than $5 \mathrm{mg}$ /day for 3 or more consecutive months. Previous fragility fracture, family history of hip fracture, current tobacco use, glucocorticoid use, chronic faller, and back pain were measured as binary variables.

Physical activity was classified as a) low, not even housework is performed, b) moderate, performs regular housework, walks irregularly, gardening, and c) high, performs regular physical activity aside from their daily routine, at least twice a week for 30 minutes [22].

To determine calcium intake, subjects were asked whether they usually drank milk and if they ate cheese or yogurt. If dairy was consumed, individuals were asked to quantify their consumption of milk or yogurt $(\mathrm{mL} / \mathrm{d})$ and cheese $(\mathrm{g} / \mathrm{d})$ during the 7 days prior to the interview.

Race was defined based on self-reported race of second-generation ancestors, an approach previously used in the Brazilian population [23]. Individuals with four grandparents reported to be Caucasian were classified as white. Individuals with both African and Caucasian ancestors (mixed race) was classified as non-Caucasian. When racial information regarding the grandparents was unavailable, an individual's race was determined by the race of his or her parents. Descendants of other races were not included.

Of the 1368 individuals recruited (866 women and 553 men), 1025 participated and blood samples were collected from each participant. Of the participants, $123(12 \%)$ were excluded for the following reasons: 70 were using drugs or supplements that affected bone metabolism (including anticonvulsants, bisphosphonates, vitamin D, and calcium), 20 had a history of previous cancer ( $<5$ years), 15 had primary hyperparathyroidism (serum calcium 
$>10.5 \mathrm{mg} / \mathrm{dL}$ and PTH $>65 \mathrm{pg} / \mathrm{mL}$ ), 12 had renal insufficiency (creatinine $>1.5 \mathrm{mg} / \mathrm{dL}$ ) and 6 had rheumatoid arthritis (Figure 1).

Subjects with grade 1 fractures $(n=133)$ were excluded from this study because evidence suggests that mild deformity is caused by either normal variation or is the result of vertebral remodeling with aging, and epidemiological studies rarely characterize mild deformities as "fractures," defining only grade 2 (moderate) or grade 3 (severe) as fractures [24-25]. Of the remaining 769 subjects, 462 were women and 307 were men. Males and females were analyzed separately, and each gender was divided into 2 groups based on whether vertebral fractures (either moderate or severe) were present. Individuals without vertebral fracture were designated in the NO FRACTURE group; subjects presenting at least one moderate or severe vertebral fracture were assigned to the FRACTURE group.

The study was approved by the Local Ethics in Research Committee of the São Paulo University, School of Medicine and all participants gave written informed consent.

\section{Anthropometry}

The height (without shoes) of each participant was measured to the nearest $0.1 \mathrm{~cm}$ with a wall-mounted stadiometer. The weight of each participant (without shoes, wearing only light clothing) was measured to the nearest $0.25 \mathrm{~kg}$ using a double-beam balance scale. Body mass index (BMI) was calculated by dividing the participants' weight (kilograms) by height squared (square meters).

\section{Laboratory evaluation}

Blood samples were collected under fasting conditions (between 8 and 10 a.m.), on the same day that VFA images were obtained, and stored at $-70^{\circ} \mathrm{C}$ for later analysis.

The serum concentrations of calcium (adjusted for the albumin concentration), phosphorus, alkaline phosphatase, creatinine and glucose were determined using standard 
automated laboratory methods. The estimated glomerular filtration rate (eGFR) was calculated using the Cockroft-Gault equation [26 - Cockcroft DW et al., 1976].

The serum concentration of 25-hydroxyvitamin D (25OHD) was measured using a radioimmunoassay technique (DiaSorin, Stillwater, MN, USA) with a lower detection limit of $5 \mathrm{ng} / \mathrm{mL}$. The intra and inter-assay variation coefficients in our laboratory were $10.5 \%$ and $17.8 \%$, respectively. Intact parathyroid hormone (iPTH) serum concentrations were measured by immunoradiometric assay (ELSA-PTH, CIS bio international, France), with reference variations of $11-65 \mathrm{pg} / \mathrm{mL}$. The variation coefficients were $2,5 \%$ and $6,1 \%$, respectively.

\section{Bone mineral density}

Bone mineral density (BMD) was measured by dual X-ray absorptiometry (DXA) using Hologic densitometry equipment (Hologic Inc. Bedford, MA, USA, Discovery model) at the following regions: lumbar spine, femoral neck, and total femur. All BMD measurements were performed by the same experienced technologist. Precision error for BMD measurements was determined based on standard International Society for Clinical Densitometry (ISCD) protocols [27]. We calculated the least significant change (LSC) with $95 \%$ confidence to be $0.033 \mathrm{~g} / \mathrm{cm}^{2}$ at the AP spine, $0.047 \mathrm{~g} / \mathrm{cm}^{2}$ at the femoral neck, and $0.039 \mathrm{~g} / \mathrm{cm}^{2}$ for the total femur.

\section{Assessment of Vertebral Fracture}

Standard lateral thoracic and lumbar spine radiographs were taken using a 40 " tubeto-film distance centered at T7 and L2. All images provided good visibility of all vertebrae from T4 to L4 and vertebrae could be reliably identified.

The identification of vertebral fractures was performed by two concomitant readers who were each experienced in this area. The readers evaluated each T4-L4 vertebrae image to decide whether it contained a fracture. Vertebral Fracture was classified using a Genant 
semiquantitative (SQ) approach [8]. Each fractured vertebra identified was classified by grade based on the Genant SQ scale, where mild (grade 1) is a reduction of $20-25 \%$ of anterior, middle, and/or posterior height, moderate (grade 2) a reduction of $26-40 \%$ in any height and severe (grade 3 ) a reduction of over $40 \%$ in any height.

\section{Statistical Analysis}

Results were expressed in mean \pm standard deviation or percentages. Differences between the two groups were evaluated using Student t-test, Mann-Whitney U or Chi square tests. Logistic regression models were used to analyze which factors were independently associated with moderate or severe vertebral fractures. Only variables significantly $(p<0.05)$ associated with moderate/severe vertebral fractures in the univariate analysis were included in the final logistic regression. Models of logistic regression were performed for each gender category. Because colinearity existed between BMD sites, sites were sequentially added to the logistic-regression and the best model was selected. Hosmer and Lemeshow tests were used to adjust the logistic-regression models. These findings are presented as adjusted odds ratios (OR) with corresponding 95\% confidence intervals $(95 \% \mathrm{CI})$. All analyses were performed using the Stata 9.0 software. 
Figure 1 - Study population.

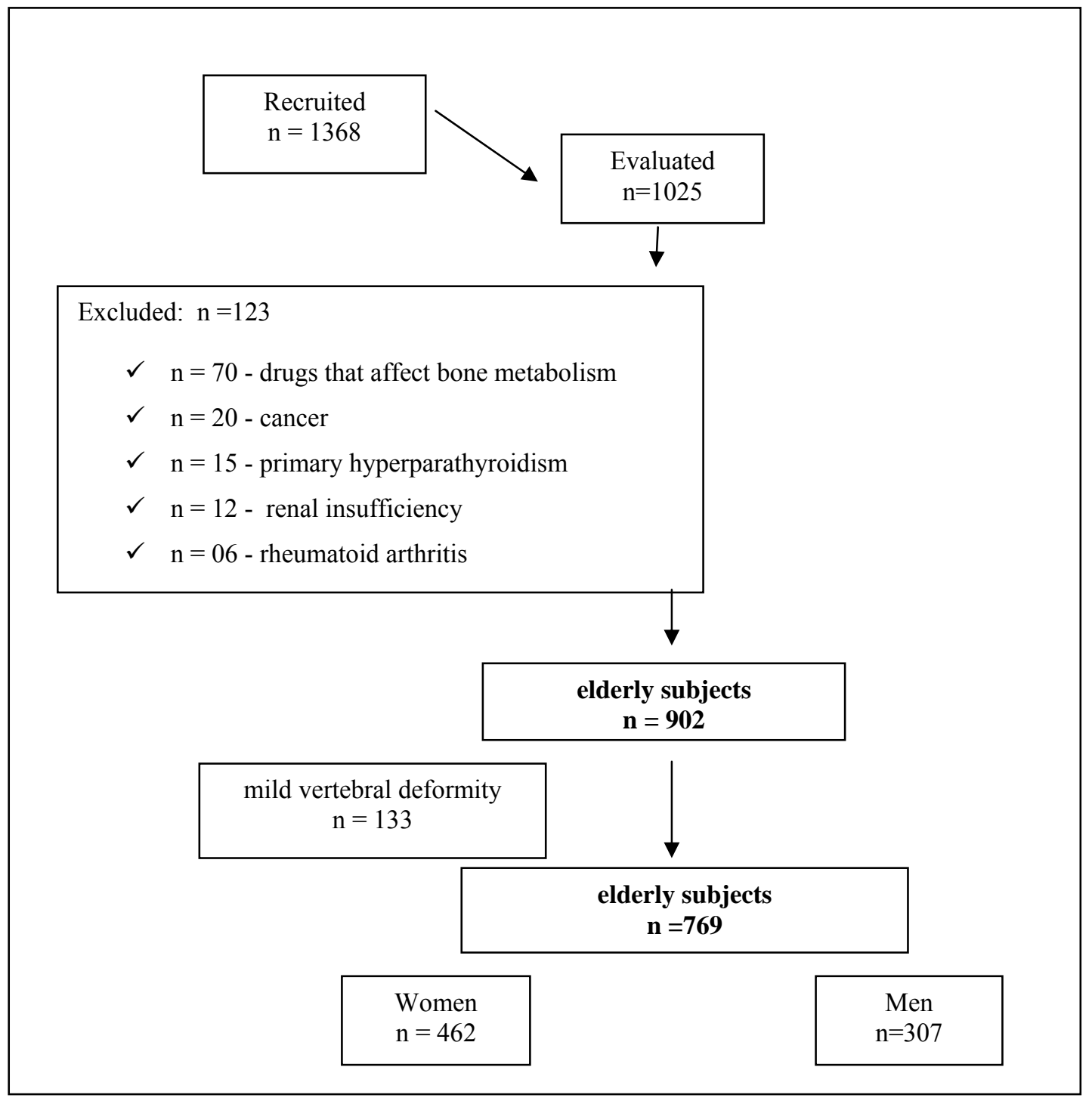




\section{Results}

Vertebral fracture was observed in $18.5 \%(95 \%$ CI 15.8-21.2) of this Brazilian population. The frequency of vertebral fracture was $16.7 \%$ (95\% CI 13.3-20.1) within women and $21.2 \%$ in men $(95 \%$ CI 16.6-25.7) $(\mathrm{P}=0.156)$. The vertebral fractures distributed by age and gender are shown in Table 1 .

\section{Female study}

Within the women in this study, individuals in the FRACTURE group were significantly older $(77.0 \pm 5.8$ vs. $72.8 \pm 4.9$ years, $p<0.001)$ and smaller $(148.3 \pm 6.0$ and $151.0 \pm 6.0 \mathrm{~cm}, \mathrm{p}<0.001)$ than women of the NO FRACTURE group (Table 2). The women in the FRACTURE group were less likely to perform high physical activity. Other previously described risk factors for osteoporosis and fractures included hypertension and diabetes, were also analyzed but the frequency of these characteristics were similar in both groups of women $(\mathrm{p}>0.05)$ (Table 2). The FRACTURE group of women showed lower eGFR $(52.5 \pm 18.5$ vs. $57.4 \pm 18.1 \mathrm{ml} / \mathrm{kg} / \mathrm{min}, \mathrm{p}=0.03)$, higher iPTH $(47.1 \pm 26.0$ vs. $41.3 \pm$ $21.5 \mathrm{pg} / \mathrm{mL}, \mathrm{p}=0,037)$ and lower $25 \mathrm{OHD}(15.5 \pm 7.3$ vs. $18.7 \pm 9.5 \mathrm{ng} / \mathrm{mL}, \mathrm{p}=0.005)$ relative to the NO FRACTURE group (Table 3).

BMD and T-scores of femoral neck and total femur were significantly lower in women within the FRACTURE group compared to women in the NO FRACTURE group $(\mathrm{p}<0.001)$, but no difference was observed between the two groups in the BMD and T-scores of the L1-L4 spine ( $>00.05)$ (Table 3).

Logistic regression analysis included age, height, high physical activity, eGFR, iPTH, 25OHD in combination with femoral neck T-score and total femur T-score in the female population reveled that age and femoral neck T-score were significantly and independently associated with the presence of moderate/severe vertebral fractures in the women (Table 4). 
Male study

Of all the measured anthropometric and clinical risk factors associated with osteoporosis and fractures, within the group of men assessed, chronic faller was the only factor significantly associated with the FRACTURE group (15.4 vs. 7.4\%, $\mathrm{p}=0.048)$ (Table 2).

No differences were found in the laboratory parameters when we compared the FRACTURE and NO FRACTURE groups within the male population (Table 3). However, BMD of femoral neck and total femur was found to be significantly lower in men in the FRACTURE group compared to the NO FRACTURE group $(\mathrm{p}<0.001)$. Likewise, the femoral neck T-score was significantly lower in men within the FRACTURE group $(\mathrm{p}<0.001)$ (Table 3).

Logistic regression analysis including these two significant variables (chronic faller and $\mathrm{T}$-score of femoral neck) demonstrated that both parameters remained significant for moderate and severe vertebral fractures in the male population (Table 5). 


\section{Discussion}

This is the first epidemiological study in Brazil designed to characterize factors associated with vertebral fractures. We assessed a variety of clinical risk factors, bone laboratory parameters and BMD of both women and men aged 65 years or older. This sample was demographically similar to the age, gender and social class distribution shown in the 2000 Brazilian census [17], and is thus likely representative of the bulk of the Brazilian elderly population.

Our study employed the Genant semiquantitative (SQ) method to diagnose vertebral fracture. This method is thought to be more objective and reproducible than other qualitative methods, with better interobserver agreement, and the SQ grading system method provides useful information in epidemiological and clinical study of osteoporosis [13]. Furthermore, this method could easily be used in clinical practice, and knowledge of normal Brazilian vertebral height is not necessary in diagnosing vertebral fracture [7].

Vertebral fractures were present in $18.6 \%$ of the total sampled population: $16.7 \%$ of women and $21.2 \%$ of men showed this type of injury. The frequency of vertebral fracture in women within our study was higher than that found in Latin American (14.7\%) and Brazil (14.2\%) in a previous LAVOS study [7]. This discrepancy may be because we assessed a somewhat older population in this study $(73.5 \pm 5.4$ vs. $68.36 \pm 10.96$ years, $\mathrm{p}<0.001)$. Indeed, comparing age distribution, the higher prevalence of vertebral fracture seen in our study was related to the elevated frequency of fracture in individuals over the age of 80 . Other possible explanations are that only the female population was assessed in the previous study, and the previous study used a different method to define vertebral fracture [7].

A European study [19] described a prevalence of vertebral fracture of $12 \%$ for women and $12.2 \%$ for men, however, the age group of that population (50-84 years) was younger than the our population (65-94 years). 
Consistent with previous studies, our study demonstrated a positive correlation between age and vertebral fracture [24], with fractures more common in older individuals. In the previous LAVOS study, the prevalence of fracture was $6.9 \%$ in women of age group 50 59 years, increasing to $27.8 \%$ in individuals over 80 years [6].

The finding that femoral neck BMD was associated with vertebral fracture in both women and men and not with lumbar spine BMD may be due to established effects of aging on spine site. Alterations associated with ageing, including extraskeletal calcification, osteophytes, and facet joint osteoarthritis in and around the lumbar spine may mask the measurement of BMD at this site [28-31].

The association of vertebral fracture with the chronic faller condition in men was somewhat surprising, although this relationship is well known for hip fractures [32-34]. Observational cohort and case-control studies imply that balance training and muscle strength improvement could reduce the risk of falling and hip fracture [35-36]. If balance improvement could reduce other fragility fractures, including vertebral fractures has to be confirmed.

It is important to emphasize that prevalent vertebral fractures are associated with an increase in morbidity and mortality [5]. Previous studies have shown an increase of $20 \%$ in mortality five years after vertebral fracture [37]. Moreover, post-menopausal women with severe vertebral fracture are at the highest risk of subsequent vertebral and nonvertebral fracture [4]. Indeed, the severity of a previous fracture was found to be a better predictor than BMD of future nonvertebral fracture risk [2].

In conclusion, vertebral fractures were a common condition in the Brazilian elderly population. Moreover, femoral neck BMD was significantly correlated with vertebral fracture in both women and men. This finding strongly supports the ISCD recommendation that vertebral assessment should be utilized in individuals older than 65 years if low BMD is present [38]. 


\section{Acknowledgments}

This work was supported by grants from: Fundação de Amparo e Pesquisa do Estado de São Paulo (FAPESP) \#03/09313-0 and \#04/12694-8; Conselho Nacional de Ciência e Tecnologia (CNPQ) \#305691/2006-6 (RMRP) and Coordenação de Aperfeiçoamento de Pessoal de Nível Superior (CAPES) (JBL).

\section{No Disclosures}




\section{REFERENCE}

1. Delmas PD, van de Langerijt L, Watts NB, Eastell R, Genant H, Grauer A et al. (2005) Underdiagnosis of Vertebral Fractures is a Worldwide Problem: The IMPACT Study Group. J Bone Miner Res 20:557-563

2. Cooper C, Atkinson EJ, O'Fallon WM, Melton LJ (1992) Incidence of clinically diagnosed vertebral fractures: A population-based study in Rochester, Minesota, 1985-1989. J Bone Miner Res 7:221-227

3. Delmas PD, Genant HK, Crans GG, Stock JL, Wong M, Siris E, Adachi JD (2003) Severity of prevalent vertebral fractures and the risk of subsequent vertebral and nonvertebral fractures: results from the MORE trial. Bone 33:522-532

4. Ismail AA, Cockerill W, Cooper C, Finn JD, Abendroth K, Parisi G et al. (2001) Prevalent vertebral deformity predicts incident hip though not distal forearm fracture: results from the European Prospective Osteoporosis Study. Osteoporos Int $12: 85-90$

5. Hasserius R, Karlsson MK, Jonsson B, Redlund-Johnell I, Johnell O (2005) Longterm morbidity and mortality after a clinically diagnosed vertebral fracture in the elderly--a 12- and 22-year follow-up of 257 patients. Calcif Tissue Int 76:235-242

6. Pinheiro MM, Ciconelli RM, Martini LA, Ferraz MB (2009) Clinical risk factors for osteoporotic fractures in Brazilian women and men: the Brazilian Osteoporosis Study (BRAZOS). Osteoporos Int. 20:399-408

7. Clark P, Cons-Molina F, Deleze M, Ragi S, Haddock L, Zanchetta JR et al. (2009) The prevalence of radiographic vertebral fractures in Latin American countries: the Latin American Vertebral Osteoporosis Study (LAVOS). Osteoporos Int 20:275-282

8. Genant HK, WuCY, van Kuijk C, Nevitt MC (1993) Vertebral fracture assessment using a semiquantitative technique. J Bone Miner Res 8:1137-1148 
9. Lentle BC, Brown JP, Khan A, Leslie WD, Levesque J, Lyons DJ et al. (2007) Recognizing and reporting vertebral fractures: reducing the risk of future osteoporotic fractures. Can Assoc Radiol J 58:27-36

10. Black DM, Reiss TF, Nevitt MC, Cauley J, Karpf D at al (1993) Design of the Fracture Intervention Trial Osteoporos Int. 3:S29-39

11. Harris ST, Watts NB, Genant HK, McKeever CD, Hangartner T, Keller M et al. (1999) Effects of risedronate treatment on vertebral and nonvertebral fractures in women with postmenopausal osteoporosis: a randomized controlled trial. Vertebral Efficacy With Risedronate Therapy (VERT) Study Group. JAMA 13;282:1344-1352

12. Siris E, Adachi JD, Lu Y, Fuerst T, Crans GG, Wong M, Harper KD, Genant HK. (2002) Effects of raloxifene on fracture severity in postmenopausal women with osteoporosis: results from the MORE study. Multiple Outcomes of Raloxifene Evaluation. Osteoporos Int 13:907-913

13. Ferrar L, Jiang G, Adams J, Eastell R (2005) Identification of vertebral fractures:un update. Osteoporis Int 16:717-728

14. Reginster JY, Seeman E., De Vernejoul MC, Adami S, Compston J, Phenekos C et al. (2005) Strontium Ranelate Reduces the Risk of Nonvertebral Fractures in Postmenopausal Women with Osteoporosis: Treatment of Peripheral Osteoporosis (TROPOS) Study. J Clin Endocrinol Metab 90:2816-2822

15. Samelson EJ, Hannan MT, Zhang Y, Genant HK, Felson DT, Kiel DP (2006) Incidence and risk factors for vertebral fracture in women and men: 25-year followup results from the population-based Framingham study.. J Bone Miner Res. 21:1207-1214

16. Instituto Brasileiro de Geografia e Estatística 2006. Censo 2000: Brazil http://www.ibge.gov.br/censo; accessed 20 March 2007 
17. Instituto Brasileiro de Geografia e Estatística - IBGE. Perfil dos Idosos Responsáveis pelos Domicílios no Brasil 2000. Estudos \& Pesquisas. Informação Sociodemográfica e Socioeconômica 9. Rio de Janeiro: IBGE, 2002

18. O'Neill TW, Cooper C, Cannata JB, Diaz Lopez JB, Hoszowski K, Johnell O et al. (1994) Reproducibility of a questionnaire on risk factors for osteoporosis in a multicentre prevalence survey: the European Vertebral Osteoporosis Study. Int J Epidemiol 23(3):559-565

19. O'Neill TW, Felsenberg D, Varlow J, Cooper C, Kanis JA, Silman AJ (1996) The prevalence of vertebral deformity in european men and women: the European Vertebral Osteoporosis Study. J Bone Miner Res 11:1010-1018

20. Seeley DG, Browner WS, Nevitt MC, Genant HK, Scott JC, Cummings SR (1991) Which fractures are associated with low appendicular bone mass in elderly women? The Study of Osteoporotic Fractures Research Group. Ann Intern Med 115:837-842

21. Schwartz AV, Villa ML, Prill M, Kelsey JA, Galinus JA, Delay RR et al. (1999) Falls in older Mexican-American womem. J Am Geriatr Soc 47:1371-1378

22. Fitti JE, KovarM (1987) The Supplement on Aging to the 1984 National Health Interview Survey. Vital Health Stat 1:1-115

23. Fuchs SC, Guimarães SM, Sortica C, Wainberg F, Dias KO, Ughini M et al. (2002) Reliability of race assessment based on the race of the ascendants: a cross-sectional study. BMC Public Health 2:1-5

24. El Maghraoui A, Mounach A, Gassim S, Ghazi M (2008) Vertebral fracture assessment in healthy men: prevalence and risk factors. Bone 43:544-548

25. Ferrar L, Jiang G, Cawthon PM, San Valentin R, Fullman R, Lambert L et al. (2007) Identification of vertebral fracture and non-osteoporotic short vertebral height in men. The MrOS study. J Bone Miner Res 1434-1441 
26. Cockcroft DW, Gault MH (1976) Prediction of creatinine clearance from serum creatinine. Nephron 16:31-41

27. Shepherd JA, Lu Y, Wilson K, Fuerst T, Genant H, Hangartner TN et al. (2006) Cross-calibration and Minimum Precision Standards for Dual-Energy X-ray Absorptiometry: The 2005 ISCD Official Positions. J Clin Densitom 9: 31-36

28. Reid IR, Evans MC, Ames R, Wattie DJ (1991) The influence of osteophytes and aortic calcification on spinal mineral density in postmenopausal women. J Clin Endocrinol Metab 72: 1372-1374

29. Masud T, Langley S, Wiltshire P, Doyle DV, Spector TD (1993) Effect of spinal osteophytosis on bone mineral density measurements in vertebral osteoporosis. BMJ 307: $172-173$

30. Muraki S, Yamamoto S, Ishibashi H, Horiuchi T, Hosoi T, Orimo H, Nakamura K (2004) Impact of degenerative spinal diseases on bone mineral density of the lumbar spine in elderly women. Osteoporos Int 15: 724-728

31. Schneider DL, Bettencourt R, Barrett-Connor E (2006) Clinical utility of spine bone density in elderly women. J Clin Densitom 9: 255-260

32. Cumming RG, Klineberg RJ (1994) Fall frequency and characteristics and the risk of hip fractures. J Am Geriatr Soc 42:774-778

33. Greenspan SL, Myers ER, Maitland LA, Resnick NM, Hayes WC (1994) Fall severity and bone mineral density as risk factors for hip fracture in ambulatory elderly. JAMA 271:128-133

34. Sherrington C, Lord SR (1998) Increased prevalence of fall risk factors in older people following hip fracture. Gerontology 44:340-344

35. Hourigan SR, Nitz JC, Brauer SG, O'Neill S, Wong J, Richardson CA (2008)

Positive effects of exercise on falls and fracture risk in osteopenic women. Osteoporos Int 19:1077-1086 
36. Karlsson MK, Nordqvist A, Karlsson C (2008) Physical activity, muscle function, falls and fractures. Food Nutr Res 52

37. Ensrud KE, Thompson DE, Cauley JA, Nevitt MC, Kado DM, Hochberg MC et al. (2000) Prevalent vertebral deformities predict mortality and hospitalization in older women with low bone mass. Fracture Intervention Trial Research Group. J Am Geriatr Soc 48:241-249

38. Schousboe JT, Vokes T, Broy SB, Ferrar L, McKiernan F, Roux C et al. (2008) Vertebral Fracture Assessment: the 2007 ISCD Official Positions. J Clin Densitom $11: 92-108$ 
Table 1. Prevalence (95\% confidence interval) of vertebral fractures by age and gender category.

\begin{tabular}{lccc}
\hline Age & ALL GENDERS & FEMALE & MALE \\
\hline $60-69$ & $12.7(8.4-17.0)$ & $4.7(1.0-8.4)$ & $22.8(14.6-31)$ \\
$70-79$ & $18.5(14.9-22.1)$ & $16.8(12.4-21.2)$ & $21.1(15.1-27.2)$ \\
$\geq 80$ & $33.7(24.0-43.4)$ & $42.6(30.2-55.0)$ & $16.1(3.2-29.1)$ \\
Overall ages & $18.5(15.8-21.2)$ & $16.7(13.3-20.1)$ & $21.2(16.6-25.7)$ \\
\hline
\end{tabular}


Table 2. Anthropometric data and risk factors for osteoporosis/fractures in individuals with moderate/severe vertebral fractures (FRACTURE group) and without fractures (NO FRACTURE group) distributed by gender category.

\begin{tabular}{|c|c|c|c|c|c|c|}
\hline & \multicolumn{3}{|c|}{ FEMALE } & \multicolumn{3}{|c|}{ MALE } \\
\hline & $\begin{array}{l}\text { FRACTURE } \\
\quad(\mathrm{n}=77)\end{array}$ & $\begin{array}{c}\text { NO } \\
\text { FRACTURE } \\
(n=385)\end{array}$ & $\mathrm{P}$ & $\begin{array}{l}\text { FRACTURE } \\
\qquad(\mathrm{n}=65)\end{array}$ & $\begin{array}{c}\text { NO } \\
\text { FRATURE } \\
(\mathrm{n}=242)\end{array}$ & $\mathrm{P}$ \\
\hline Age (years) & $77.0 \pm 5.8$ & $72.8 \pm 4.9$ & $<0.001$ & $72.8 \pm 5.0$ & $72.7 \pm 5.0$ & 0.979 \\
\hline Weight (kg) & $63.9 \pm 14.3$ & $65.7 \pm 13.3$ & 0.324 & $72.8 \pm 13.5$ & $70.1 \pm 12.0$ & 0.116 \\
\hline Height $(\mathrm{cm})$ & $148.3 \pm 6.0$ & $151.0 \pm 6.0$ & $<0.001$ & $163.4 \pm 8.2$ & $162.5 \pm 7.4$ & 0.418 \\
\hline Body mass index $\left(\mathrm{kg} / \mathrm{m}^{2}\right)$ & $29.0 \pm 5.9$ & $28.7 \pm 5.3$ & 0.686 & $27.2 \pm 4.5$ & $26.5 \pm 3.7$ & 0.162 \\
\hline Caucasian, n (\%) & $54(70.1)$ & $250(64.9)$ & 0.380 & $44(67.7)$ & $168(69.4)$ & 0.789 \\
\hline Menopause age, years & $44.6 \pm 13.3$ & $46.4 \pm 10.4$ & 0.183 & NA & NA & \\
\hline Alcohol use, n (\%) & $1(1.3)$ & $1(0.3)$ & 0.202 & $10(15.4)$ & $22(9.1)$ & 0.140 \\
\hline Current tabaco, n (\%) & $4(5.2)$ & $42(10.9)$ & 0.126 & $10(15.4)$ & $37(15.3)$ & 0.985 \\
\hline Glucocorticoid use, n (\%) & $3(3.9)$ & $5(1.3)$ & 0.111 & $2(3.1)$ & $1(0.4)$ & 0.053 \\
\hline Fragility fracture, n (\%) & $16(20.8)$ & $54(14.0)$ & 0.131 & $4(6.2)$ & $14(5.8)$ & 0.911 \\
\hline $\begin{array}{l}\text { Family history of fragility } \\
\text { fracture, } \mathrm{n}(\%)\end{array}$ & $3(4.4)^{\mathrm{a}}$ & $39(11.02)^{b}$ & 0.090 & $6(10.9)^{\mathrm{c}}$ & $12(5.5)^{\mathrm{d}}$ & 0.149 \\
\hline Chronic faller, n (\%) & $14(18.2)$ & $63(16.4)$ & 0.696 & $10(15.4)$ & $18(7.4)$ & 0.048 \\
\hline Dietary calcium intake, $\mathrm{mg} /$ dia & $425 \pm 326$ & $449 \pm 309$ & 0.541 & $408 \pm 333$ & $410 \pm 317$ & 0.965 \\
\hline High physical activity, n (\%) & $8(10.4)$ & $96(24.9)$ & 0.005 & $10(15.4)$ & $52(21.5)$ & 0.277 \\
\hline Back pain, n (\%) & $44(57.1)$ & $209(54.3)$ & 0.646 & $18(27.7)$ & $73(30.2)$ & 0.698 \\
\hline Hypertension, n (\%) & $50(64.9)$ & $289(75.1)$ & 0.066 & $36(55.4)$ & $130(53.7)$ & 0.811 \\
\hline Diabetes mellitus, n(\%) & $17(22.1)$ & $97(25.2)$ & 0.563 & $8(12.3)$ & $41(16.9)$ & 0.365 \\
\hline
\end{tabular}

Data are expressed as percentage and mean \pm standard deviation ${ }^{a} n=69,{ }^{b} n=354 \quad{ }^{c} n=22,{ }^{d} n=308$

NA- not applicable 
Table 3 - Laboratorial parameters and Bone mineral density (BMD) in individuals with moderate/severe vertebral fractures (FRACTURE group) and without fractures (NO FRACTURE group) distributed by gender category.

\begin{tabular}{|c|c|c|c|c|c|c|}
\hline & \multicolumn{3}{|c|}{ FEMALE } & \multicolumn{3}{|c|}{ MALE } \\
\hline & $\begin{array}{l}\text { FRACTURE } \\
\qquad(\mathrm{n}=77)\end{array}$ & $\begin{array}{c}\text { NO } \\
\text { FRACTURE } \\
(n=385)\end{array}$ & $\mathrm{P}$ & $\begin{array}{l}\text { FRACTURE } \\
\qquad(\mathrm{n}=65)\end{array}$ & $\begin{array}{c}\text { NO } \\
\text { FRACTURE } \\
(n=242)\end{array}$ & $\mathrm{P}$ \\
\hline Calcium, mg/dL & $9.4 \pm 0.4$ & $9.4 \pm 0.5$ & 0.500 & $9.2 \pm 0.4$ & $9.3 \pm 0.5$ & 0.171 \\
\hline Phosphorus, mg/dL & $3.6 \pm 0.5$ & $3.5 \pm 0.5$ & 0.242 & $3.2 \pm 0.6$ & $3.1 \pm 0.5$ & 0.262 \\
\hline $\begin{array}{l}\text { Alkaline } \\
\text { phosphatase, U/L }\end{array}$ & $200.8 \pm 85.7$ & $190.7 \pm 54.7$ & 0.184 & $167.5 \pm 48.9$ & $171.9 \pm 68.1$ & 0.628 \\
\hline Creatinine, $\mathrm{mg} / \mathrm{dL}$ & $0.96 \pm 0.2$ & $0.95 \pm 0.2$ & 0.598 & $1.14 \pm 0.3$ & $1.17 \pm 0.2$ & 0.569 \\
\hline $\mathrm{eGFR}, \mathrm{mL} / \mathrm{min}$ & $52.5 \pm 18.5$ & $57.4 \pm 18.1$ & 0.030 & $62.4 \pm 18.8$ & $58.5 \pm 17.6$ & 0.121 \\
\hline Glicemy, mg/dL & $113.6 \pm 26.6$ & $121.6 \pm 56.2$ & 0.225 & $112.8 \pm 41.1$ & $113.7 \pm 30.1$ & 0.840 \\
\hline iPTH, pg/mL & $47.1 \pm 26.0$ & $41.3 \pm 21.5$ & 0.037 & $37.5 \pm 16.8$ & $37.8 \pm 18.5$ & 0.914 \\
\hline 25OHD, ng/dL & $15.5 \pm 7.3$ & $18.7 \pm 9.5$ & 0.005 & $22.3 \pm 11.3$ & $21 \pm 9.1$ & 0.318 \\
\hline L1-L4 BMD, g/cm ${ }^{2}$ & $0.799 \pm 0.204$ & $0.840 \pm 0.169$ & 0.057 & $0.986 \pm 0.181$ & $1.015 \pm 0.200$ & 0.286 \\
\hline L1-L4 T-score & $-2.5 \pm 1.8$ & $-2.2 \pm 1.5$ & 0.092 & $-1.2 \pm 1.7$ & $-0.9 \pm 1.8$ & 0.318 \\
\hline $\begin{array}{l}\text { Femoral neck BMD, } \\
\mathrm{g} / \mathrm{cm}^{2}\end{array}$ & $0.600 \pm 0.123$ & $0.672 \pm 0.122$ & $<0.001$ & $0.719 \pm 0.133$ & $0.764 \pm 0.146$ & 0.026 \\
\hline $\begin{array}{l}\text { Femoral neck T- } \\
\text { score }\end{array}$ & $-2.3 \pm 1.0$ & $-1.7 \pm 1.0$ & $<0.001$ & $-1.8 \pm 1.0$ & $-1.4 \pm 1.1$ & 0.038 \\
\hline $\begin{array}{l}\text { Total femur BMD, } \\
\mathrm{g} / \mathrm{cm}^{2}\end{array}$ & $0.716 \pm 0.142$ & $0.804 \pm 0.132$ & $<0.001$ & $0.895 \pm 0.133$ & $0.940 \pm 0.154$ & 0.030 \\
\hline Total femur T-score & $-1.9 \pm 1.1$ & $-1.2 \pm 1.01$ & $<0.001$ & $-1.1 \pm 0.9$ & $-0.8 \pm 1.0$ & 0.051 \\
\hline
\end{tabular}

Data are expressed as mean \pm standard deviation

$\mathrm{eGFR}=$ estimated glomerular filtration rate, $\mathrm{iPTH}=$ intact parathyroid hormone, $25 \mathrm{OHD}=25$ hydroxivitamin D 
Table 4 - Logistic regression analysis for the presence of moderate/severe vertebral fractures in women.

\begin{tabular}{lccc} 
& OR & $95 \%$ & P \\
& adjusted & Confidence Interval & \\
\hline Age & 1.12 & $(1.06-1.18)$ & $<0.001$ \\
Femoral neck T-score & 0.64 & $(0.46-0.88)$ & 0.006 \\
\hline
\end{tabular}

OR adjusted for height, high physical activity, estimated glomerular filtration rate, intact parathyroid hormone and 25 hydroxyvitamin D

Table 5 - Logistic regression analysis for the presence of moderate/severe vertebral fractures in men.

\begin{tabular}{lccc}
\hline & OR & $95 \%$ & \\
& adjusted & Confidence Interval & \\
\hline Femoral neck T-score & 0.72 & $(0.53-0.96)$ & 0.025 \\
Chronic faller & 2.54 & $(1.1-5.9)$ & 0.031 \\
\hline
\end{tabular}

